\title{
Development of MADByTE: an NMR-Based Platform for the Direct Structural Annotation of Complex Natural Products Mixtures
}

Joseph M. Egan, Jeffrey A. van Santen, Dennis Y. Liu, Roger G. Linington*

\section{Supporting Information}

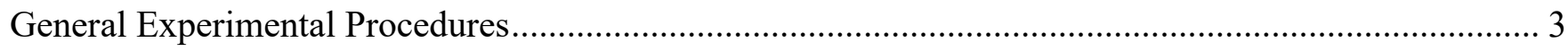

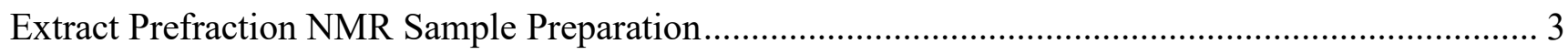

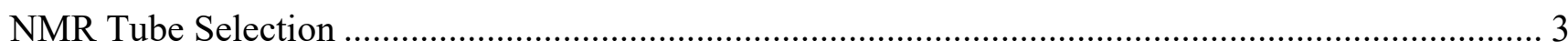

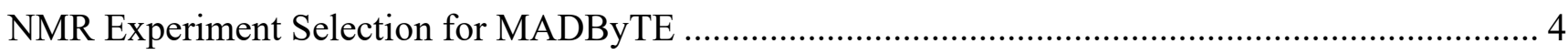

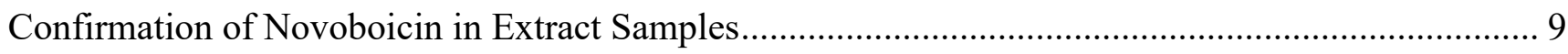

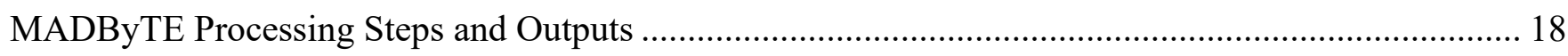

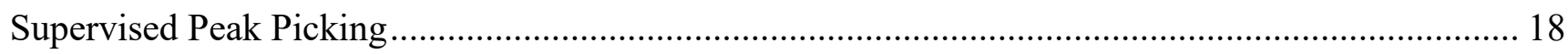

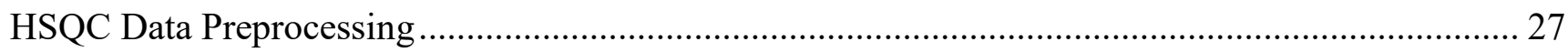

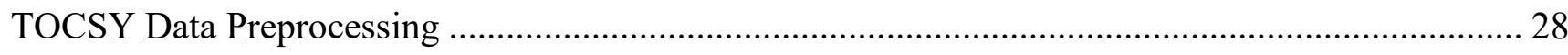

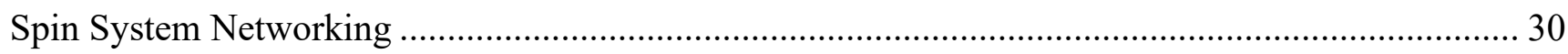

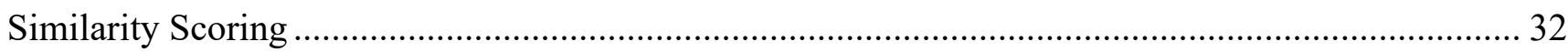

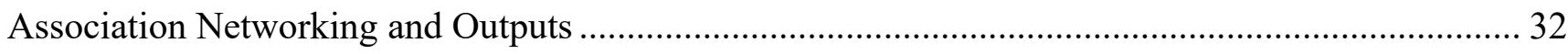

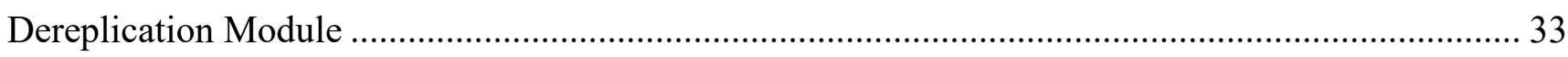

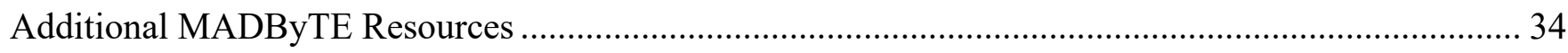

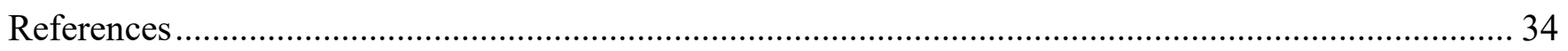




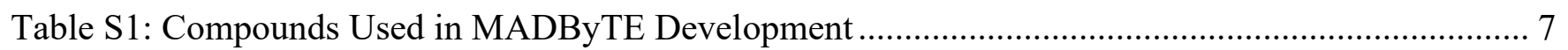

Table S2:MADByTE GUI Parameters Used for Standard Compounds ...................................................... 8

Table S3:MADByTE GUI Parameters for Prefractions Spiked With Pure Compounds............................. 9

Table S4: MADByTE GUI Parameters for 85 Extract Prefractions and Standards .................................... 9

Table S5: MADByTE GUI Parameters for Biological Activity Layering Network ................................. 9

Table S6:Bacterial Strains and Growth Conditions for Biological Evaluation ....................................... 17

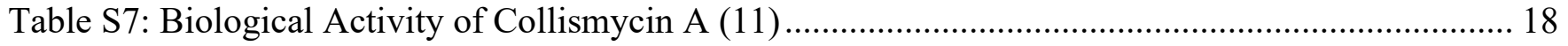

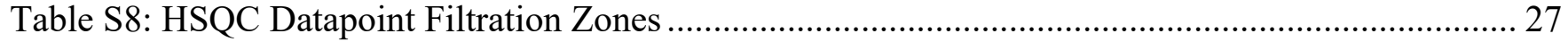

Figure S1: Glass Pipette Setup for Accurate and Reproducible Transfer of NMR Solvents ..................... 3

Figure S2: Comparison of Peak Area of $0.42 \mathrm{mg}$ of Mupirocin in a $3 \mathrm{~mm}$ Tube vs $5 \mathrm{~mm}$ Shigemi Tube in

a 5mm TCI Probe ......................................................................................................................... 4

Figure S3: Sensitivity of TOCSY vs HSQC-TOCSY for a Spin System of Lincomycin at $3.16 \mathrm{ppm}$...... 5

Figure S4: Structures of Compounds Used for MADByTE Development................................................ 6

Figure S5: Full Annotation Network of Standard Compounds Used for MADByTE Development ......... 8

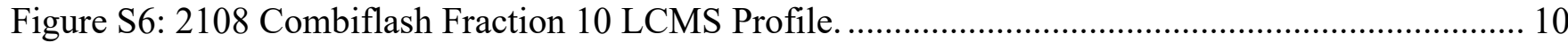

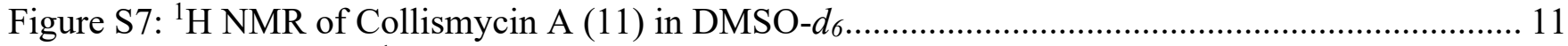

Figure S8: Expansion of ${ }^{1} \mathrm{H}$ NMR In Aromatic Region of Collismycin A (11) in DMSO- $d_{6} \ldots \ldots \ldots \ldots \ldots . . . . . .12$

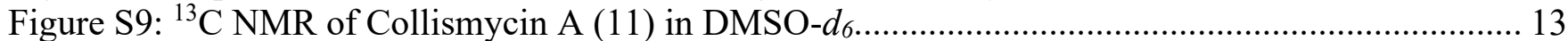

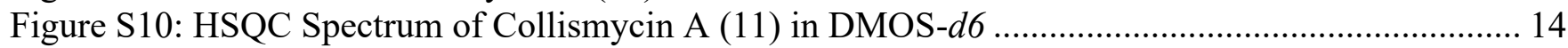

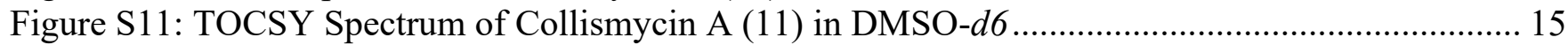

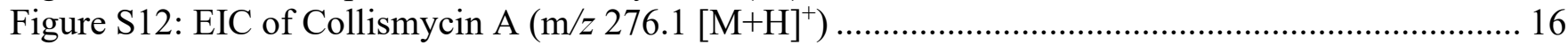

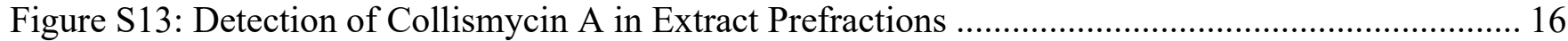

Figure S14: HSQC of Prefraction 1814E Spiked with Erythromycin ..................................................... 19

Figure S15: TOCSY of Prefraction 1814E Spiked with Erythromycin.................................................. 20

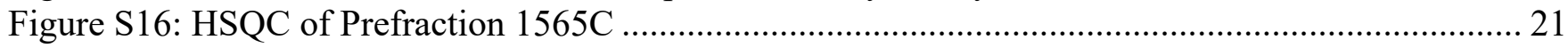

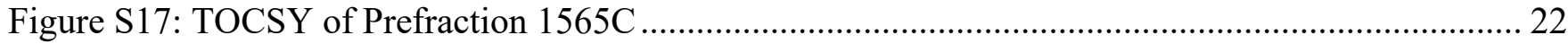

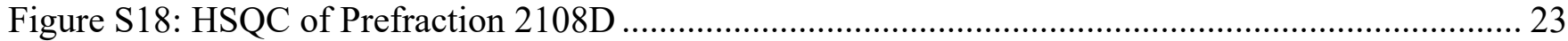

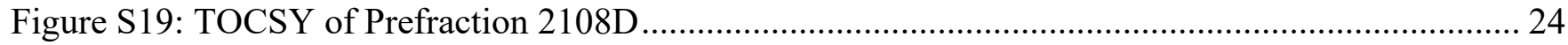

Figure S20: TOCSY of Prefraction 2108D Using Automatic Peak Picking …………........................... 25

Figure S21: HSQC of Prefraction 2108D Using Automatic Peak Picking............................................. 26

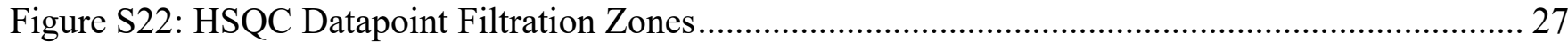

Figure S23: Typical FT vs Covariance Processing for TOCSY Spectra ............................................... 28

Figure S24: Distribution of Estimated Chemical Shifts in NP Atlas Molecules....................................... 29

Figure S25:TOCSY Data Point Filtration and Alignment Process ......................................................... 30

Figure S26: Spin System Construction from TOCSY Data of Erythromycin ........................................... 31

Figure S27:Assigment of HSQC ${ }^{13} \mathrm{C}$ Values to TOCSY Spin System Features ....................................... 31

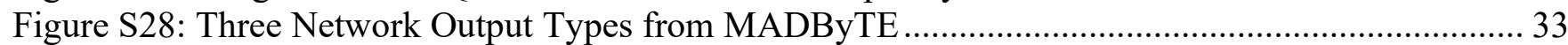

Figure S29: MADByTE Bioactivity Color Coding Example ……………………................................. 33 


\section{General Experimental Procedures}

\section{Extract Prefraction NMR Sample Preparation}

$25 \mu \mathrm{L}$ aliquots of extract prefractions were retrieved from our previously described actinobacterial library, ${ }^{1}$ dried via lyophilization, resuspended in $300 \mu \mathrm{L}$ of DMSO-d6 and lyophilized again to remove non-deuterated DMSO from the sample. Sample aliquots translated to a variable mass between 4-15 mg of dry material. Samples were dissolved in $320 \mu \mathrm{L}$ of DMSO-d6, sonicated, and $280 \mu \mathrm{L}$ were placed into a matched Shigemi tube for acquisition with care taken to ensure no solid particulate was transferred. Glass pipettes were pulled to greater length and attached to a $1000 \mu \mathrm{L}$ micropipette for accurate solvent dispensing and transfer (Figure S1). Shigemi tubes were placed under high vacuum for 30 minutes prior to use to remove water vapor, and back filled with argon.

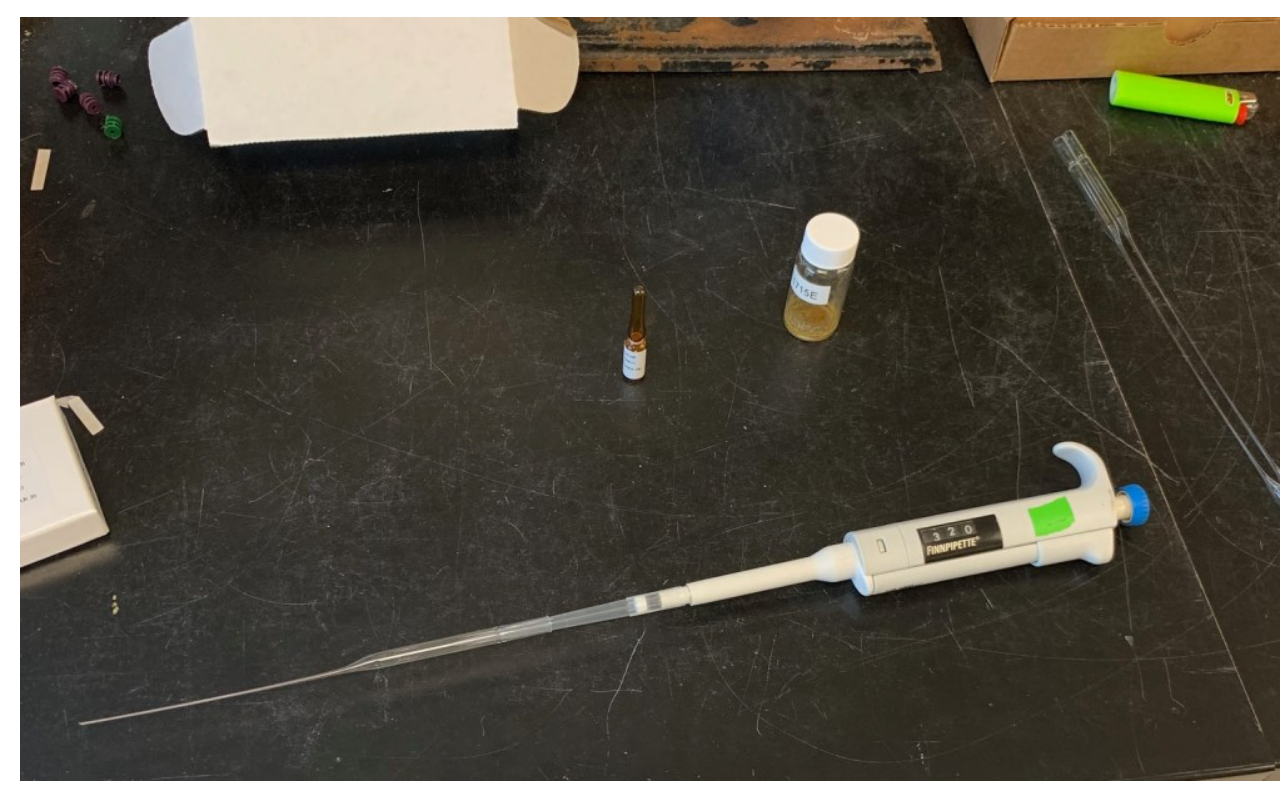

Figure S1: Glass Pipette Setup for Accurate and Reproducible Transfer of NMR Solvents

\section{$\underline{\text { NMR Tube Selection }}$}

To select the optimal tube type for obtaining spectra with good peak shape and sensitivity in a $5 \mathrm{~mm}$ probe, a sample of $0.84 \mathrm{mg}$ of mupirocin was suspended at $1 \mathrm{mg} / \mathrm{mL}$ in methanol, sonicated, and $450 \mu \mathrm{L}$ was transferred to each of two separate vials. Each vial was concentrated to dryness under a stream of nitrogen gas, resuspended in either $150 \mu \mathrm{L}$ or $250 \mu \mathrm{L}$ of DMSO-d6, and placed into a $3 \mathrm{~mm}$ NMR tube and a matched Shigemi tube, respectively. After automatic shimming using 'topshim', the $5 \mathrm{~mm}$ Shigemi tube showed an improvement in shimming time, compared to the $3 \mathrm{~mm}$ tube, which required manual shim correction. The $5 \mathrm{~mm}$ tube also afforded higher peak area and improved signal to noise ratio compared to the $3 \mathrm{~mm}$ tube (Figure S2). s/n was calculated using the Bruker command SINO with the signal region between $3.98-4.02 \mathrm{ppm}$ and the noise region defined as $7-8 \mathrm{ppm}$ with an s/n ratio of 5953.91 for the 3 $\mathrm{mm}$ tube and 14181.04 for the $5 \mathrm{~mm}$ Shigemi tube. All experiment settings were kept consistent for both experiments. 


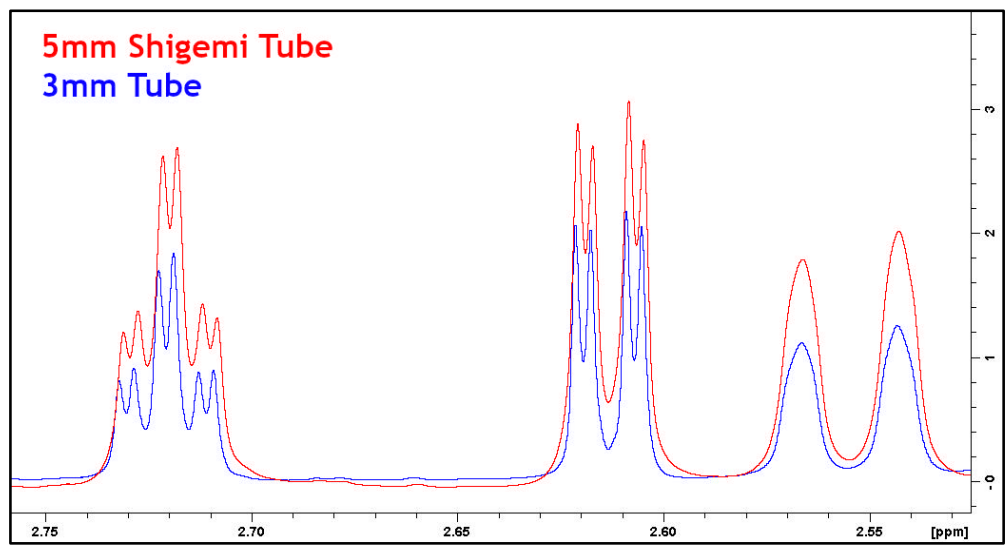

Figure S2: Comparison of Peak Area of $0.42 \mathrm{mg}$ of Mupirocin in a $3 \mathrm{~mm}$ Tube vs $5 \mathrm{~mm}$ Shigemi Tube in a 5 mm TCI Probe

Optimizing conditions and increasing the volume of solvent to $280 \mu \mathrm{L}$ in the $5 \mathrm{~mm}$ Shigemi tube allowed for easy automatic shimming. An additional benefit was the ability to use Shigemi tubes in an autosampler to increase sample throughput, as opposed to manual loading and shimming required by the $3 \mathrm{~mm}$ tube setup.

\section{NMR Experiment Selection for MADByTE}

MADByTE is structured to use two separate experiments, the HSQC and TOCSY. HSQC variants include the HSQC-TOCSY experiment, where TOCSY cross peaks are arrayed on the ${ }^{13} \mathrm{C}$ axis in the $\mathrm{F} 1$ dimension. In principle, a combination HSQC-TOCSY offers time savings, as only one experiment must be acquired. To evaluate the usefulness of the combined HSQC-TOCSY, $8 \mathrm{mg}$ of lincomycin was dissolved in $500 \mu \mathrm{L}$ of DMSO- $d_{6}$ and subjected to HSQC-TOCSY analysis. Using 16 scans in the HSQCTOCSY, the sensitivity loss was noticeable when compared to a TOCSY using only 2 scans (Figure S3). $\mathrm{S} / \mathrm{n}$ was calculated for these slices with $\mathrm{s} / \mathrm{n}$ of 676.3 for TOCSY at $2 \mathrm{scans}$, compared to a s/n of 160.9 for the HSQC-TOCSY. As MADByTE was designed for the investigation of crude mixtures where some metabolites are present in low abundance, we elected to acquire the individual HSQC and TOCSY spectra for this study.

By requiring the HSQC as a separate experiment, explicit ${ }^{1} \mathrm{H}^{13} \mathrm{C}$ relationships can be assigned. This is not possible in the HSQC-TOCSY. As an added benefit of using orthogonal experiments rather than a singular experiment, MADByTE requires resonances in both the HSQC and TOCSY to be present in order to be counted as a valid resonance, ensuring artifacts from noise in one spectrum does not confound the results of the combination processing. 


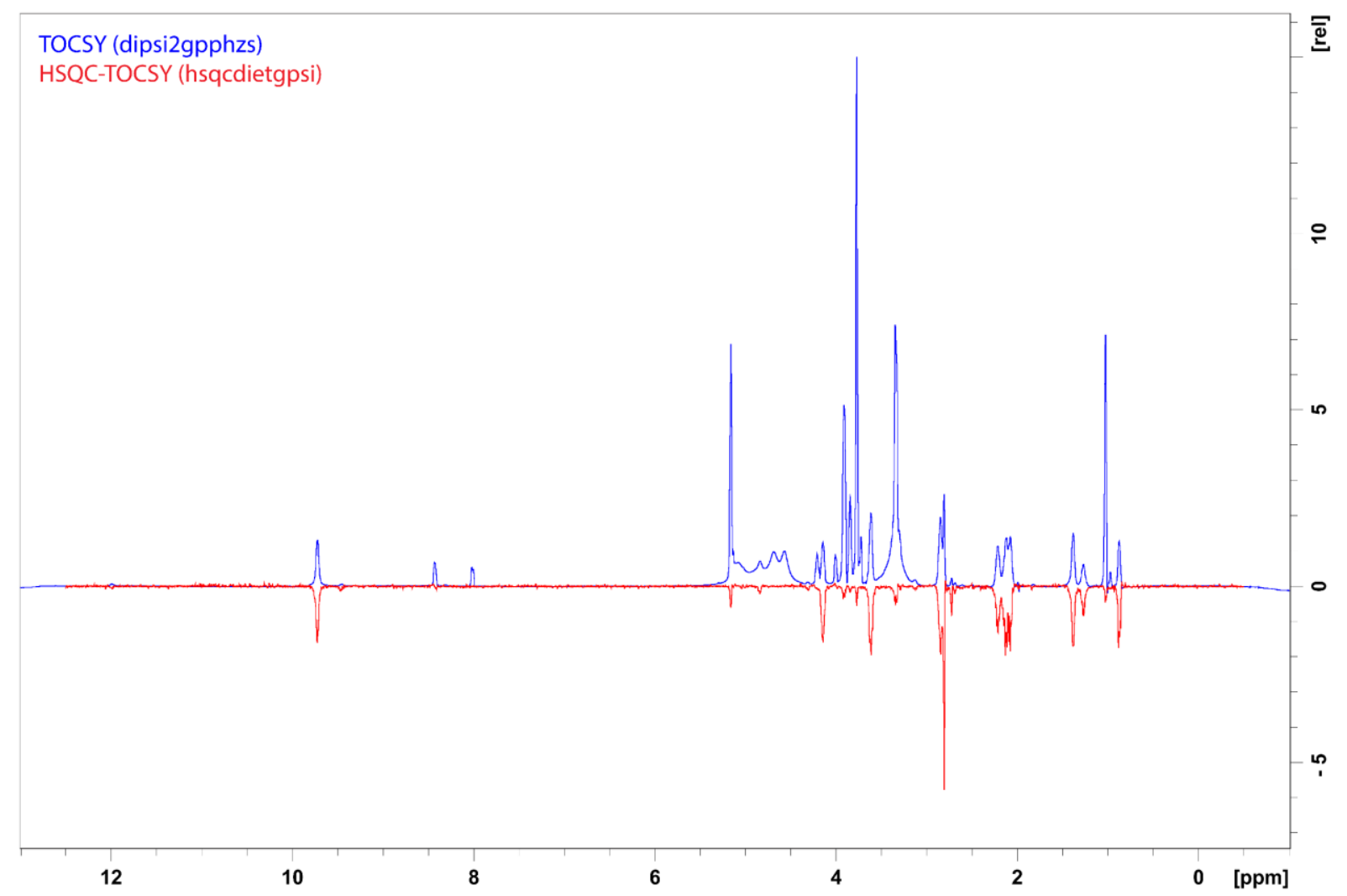

Figure S3: Sensitivity of TOCSY vs HSQC-TOCSY for a Spin System of Lincomycin at 3.16 ppm 

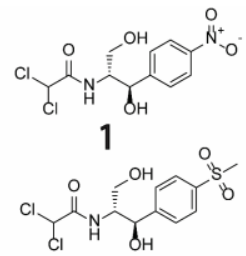

2
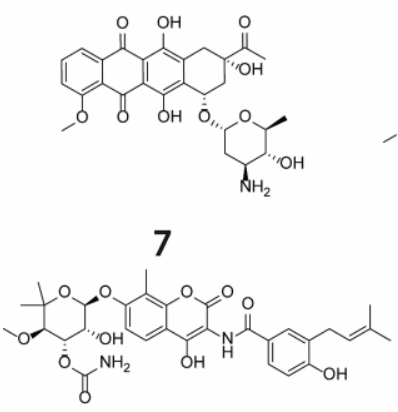

10

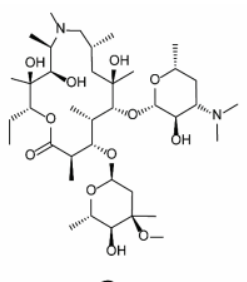

3
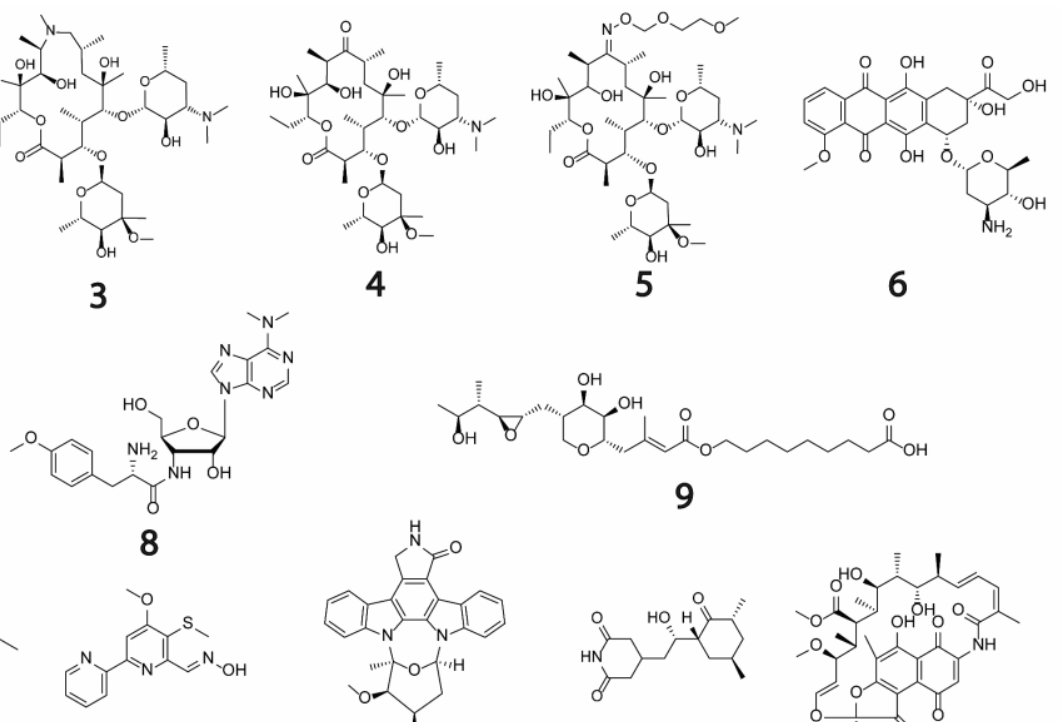

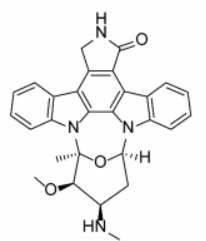

12

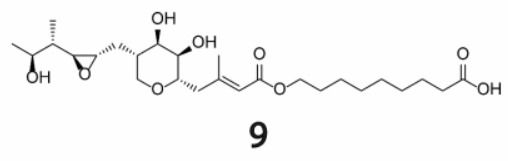

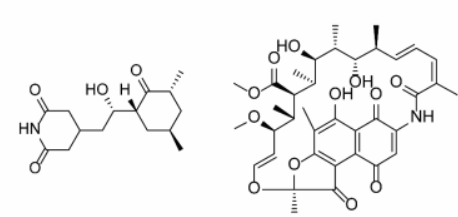

13

14

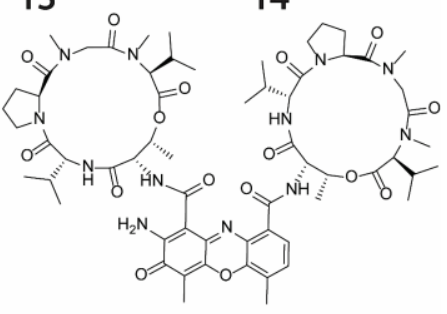

18

15

16

17

Figure S4: Structures of Compounds Used for MADByTE Development 
Table S1: Compounds Used in MADByTE Development

\begin{tabular}{|c|c|}
\hline $\begin{array}{c}\text { Compound Number } \\
\text { (Figures S4,S5) }\end{array}$ & Compound \\
\hline $\mathbf{1}$ & Chloramphenicol \\
\hline $\mathbf{2}$ & Thiamphenicol \\
\hline $\mathbf{3}$ & Azithromycin \\
\hline $\mathbf{4}$ & Erythromycin \\
\hline $\mathbf{5}$ & Roxithromycin \\
\hline $\mathbf{6}$ & Epirubicin \\
\hline $\mathbf{7}$ & Daunomycin \\
\hline $\mathbf{8}$ & Puromycin \\
\hline $\mathbf{9}$ & Mupirocin \\
\hline $\mathbf{1 0}$ & Novobiocin \\
\hline $\mathbf{1 1}$ & Collismycin A \\
\hline $\mathbf{1 2}$ & Staurosporine \\
\hline $\mathbf{1 3}$ & Cycloheximide \\
\hline $\mathbf{1 4}$ & Rifamycin S \\
\hline $\mathbf{1 5}$ & Betulinic acid \\
\hline $\mathbf{1 6}$ & Ursolic acid \\
\hline $\mathbf{1 8}$ & Oleanolic acid \\
\hline & Actinomycin D \\
\hline
\end{tabular}




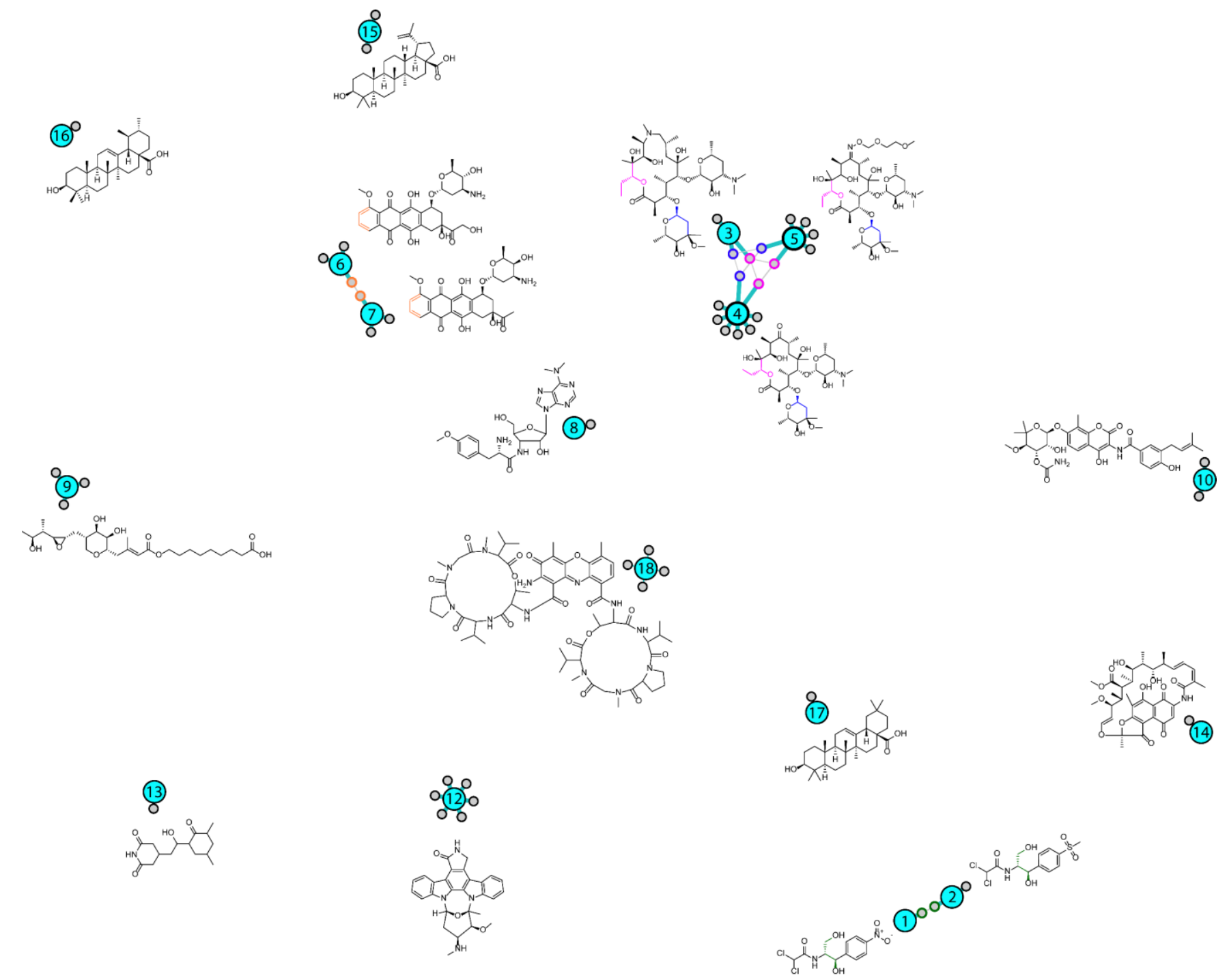

Figure S5: Full Annotation Network of Standard Compounds Used for MADByTE Development

Table S2:MADByTE GUI Parameters Used for Standard Compounds

\begin{tabular}{|c|c|}
\hline Parameter & Value \\
\hline Hppm Error & 0.05 \\
\hline Cppm Error & 0.40 \\
\hline Consensus Error & 0.03 \\
\hline Similarity Ratio & 0.51 \\
\hline Merge Multiplets & True \\
\hline Maximum Spin System Size & 40 \\
\hline
\end{tabular}


Table S3:MADByTE GUI Parameters for Prefractions Spiked With Pure Compounds

\begin{tabular}{|c|c|}
\hline Parameter & Value \\
\hline Hppm Error & 0.05 \\
\hline Cppm Error & 0.40 \\
\hline Consensus Error & 0.03 \\
\hline Similarity Ratio & 0.30 \\
\hline Merge Multiplets & True \\
\hline Maximum Spin System Size & 40 \\
\hline
\end{tabular}

Table S4: MADByTE GUI Parameters for 85 Extract Prefractions and Standards

\begin{tabular}{|c|c|}
\hline Parameter & Value \\
\hline Hppm Error & 0.05 \\
\hline Cppm Error & 0.40 \\
\hline Consensus Error & 0.03 \\
\hline Similarity Ratio & 0.30 \\
\hline Merge Multiplets & True \\
\hline Maximum Spin System Size & 40 \\
\hline
\end{tabular}

Table S5: MADByTE GUI Parameters for Biological Activity Layering Network

\begin{tabular}{|c|c|}
\hline Parameter & Value \\
\hline Hppm Error & 0.05 \\
\hline Cppm Error & 0.40 \\
\hline Consensus Error & 0.03 \\
\hline Similarity Ratio & 0.50 \\
\hline Merge Multiplets & True \\
\hline Maximum Spin System Size & 20 \\
\hline
\end{tabular}

\section{Confirmation of Novoboicin in Extract Samples}

MADByTE analysis of 85 extracts and standard compounds yielded node connections between prefractions 1565C, 1565D, and novobiocin (10). Manual investigation of the connected nodes and review of the TOCSY and HSQC spectra identified shared cross peaks as expected. Although the spectra are of varying complexity, spin system features between these samples were successfully identified as belonging to a single shared metabolite. To confirm the presence of novobiocin unequivocally, UPLC-HRMS/MS 
analysis was conducted on each prefraction against a novobiocin standard. Samples were prepared in 50:50 MeOH: $\mathrm{H}_{2} \mathrm{O}$ at $0.5 \mathrm{mg} / \mathrm{mL}$ for extract prefractions and $0.2 \mathrm{mg} / \mathrm{mL}$ for novobiocin.
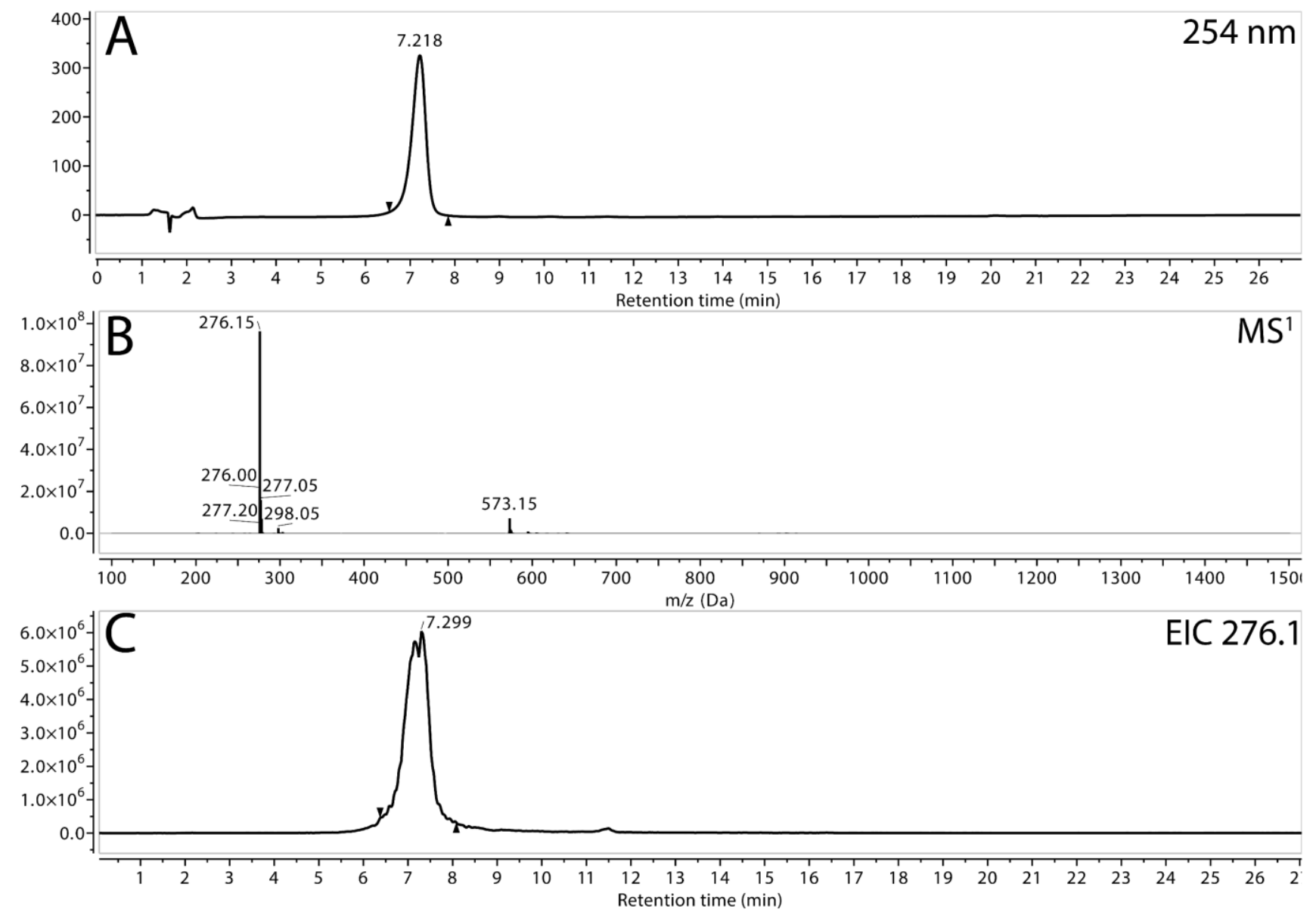

Figure S6: 2108 Combiflash Fraction 10 LCMS Profile. 


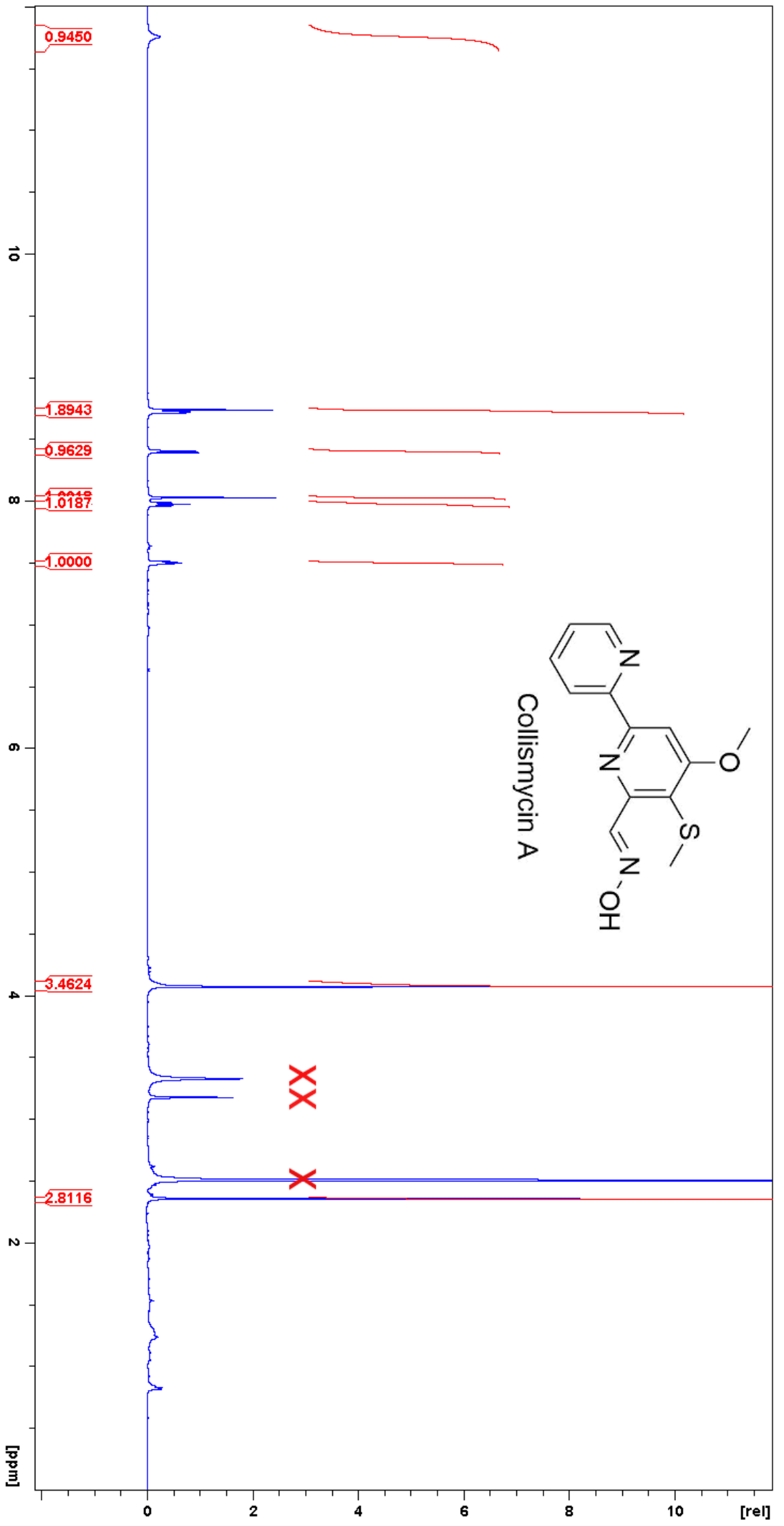

Figure S7: ${ }^{1} \mathrm{H}$ NMR of Collismycin A (11) in DMSO-d 6 


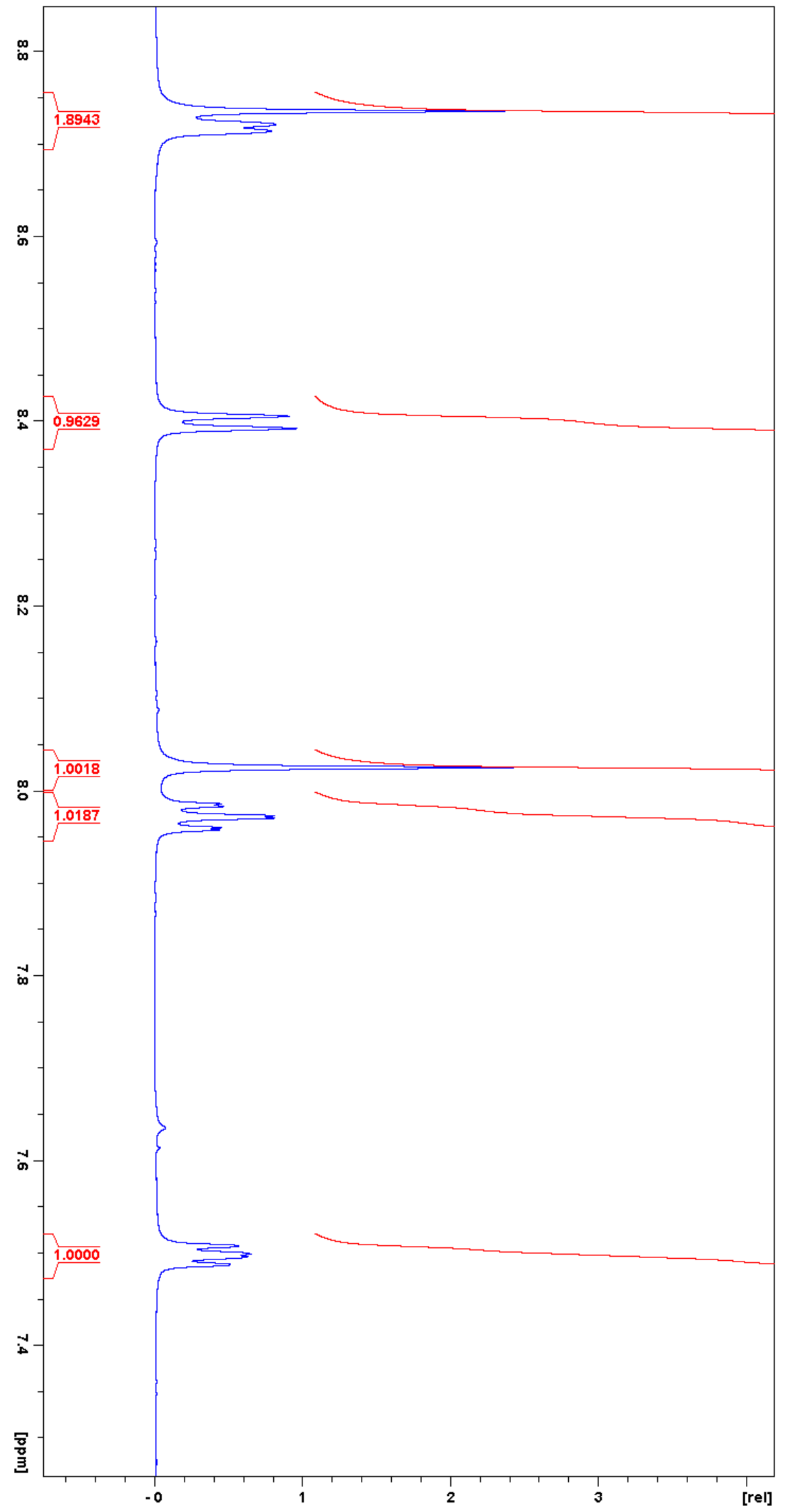

Figure S8: Expansion of ${ }^{1} \mathrm{H}$ NMR In Aromatic Region of Collismycin A (11) in DMSO-d 


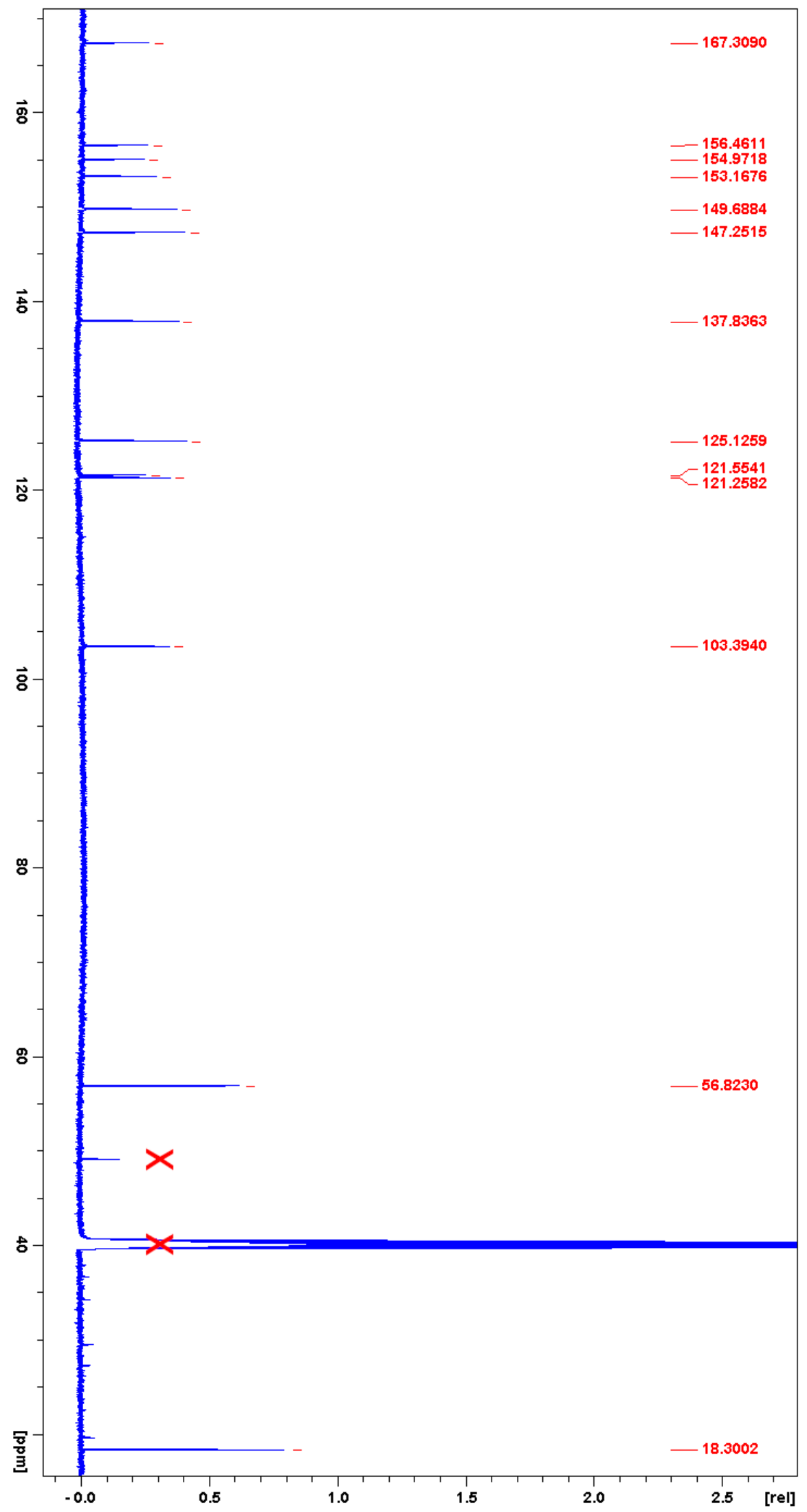

Figure S9: ${ }^{13} \mathrm{C}$ NMR of Collismycin A (11) in DMSO-d 


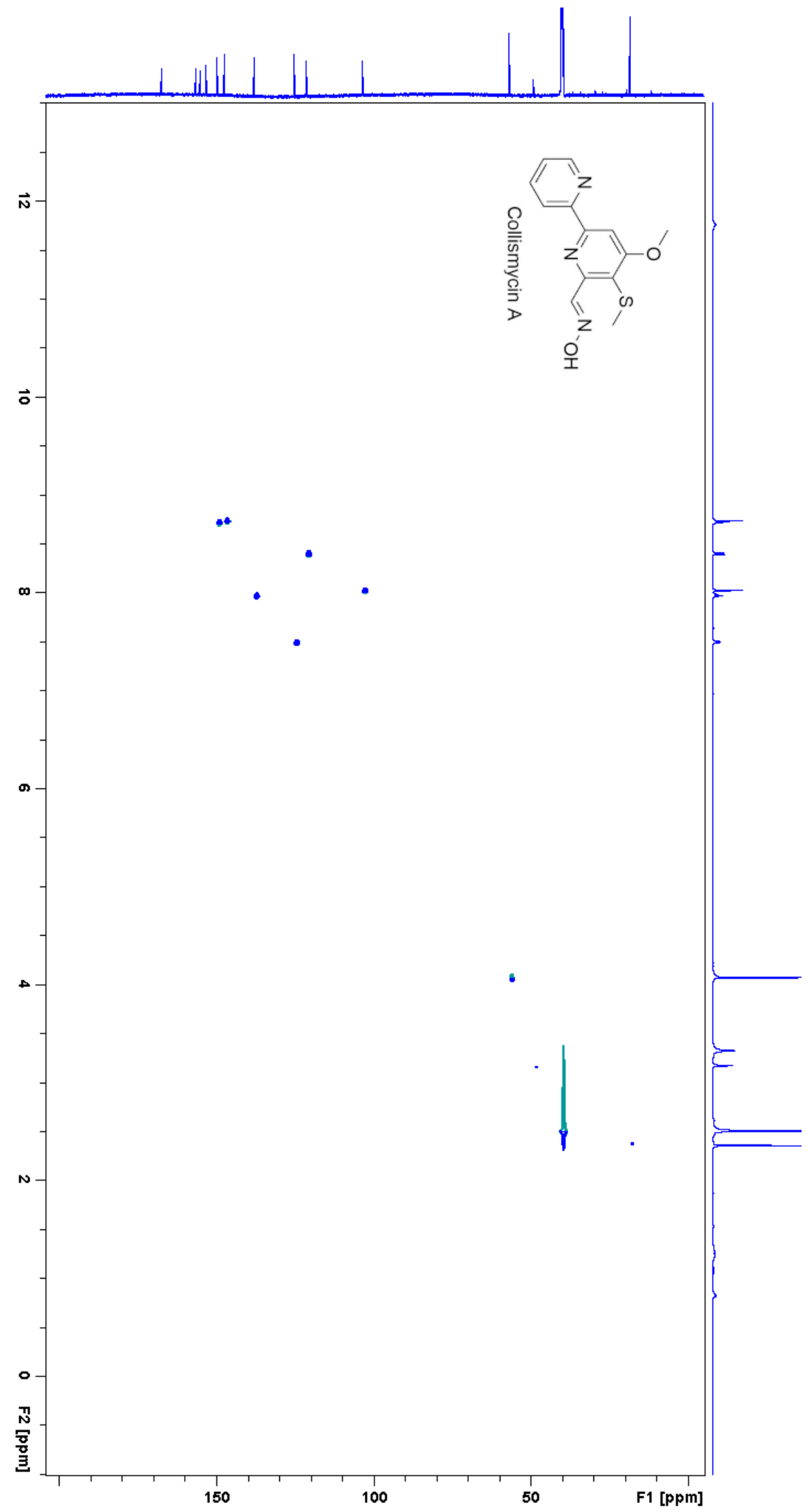

Figure S10: HSQC Spectrum of Collismycin A (11) in DMOS-d6 


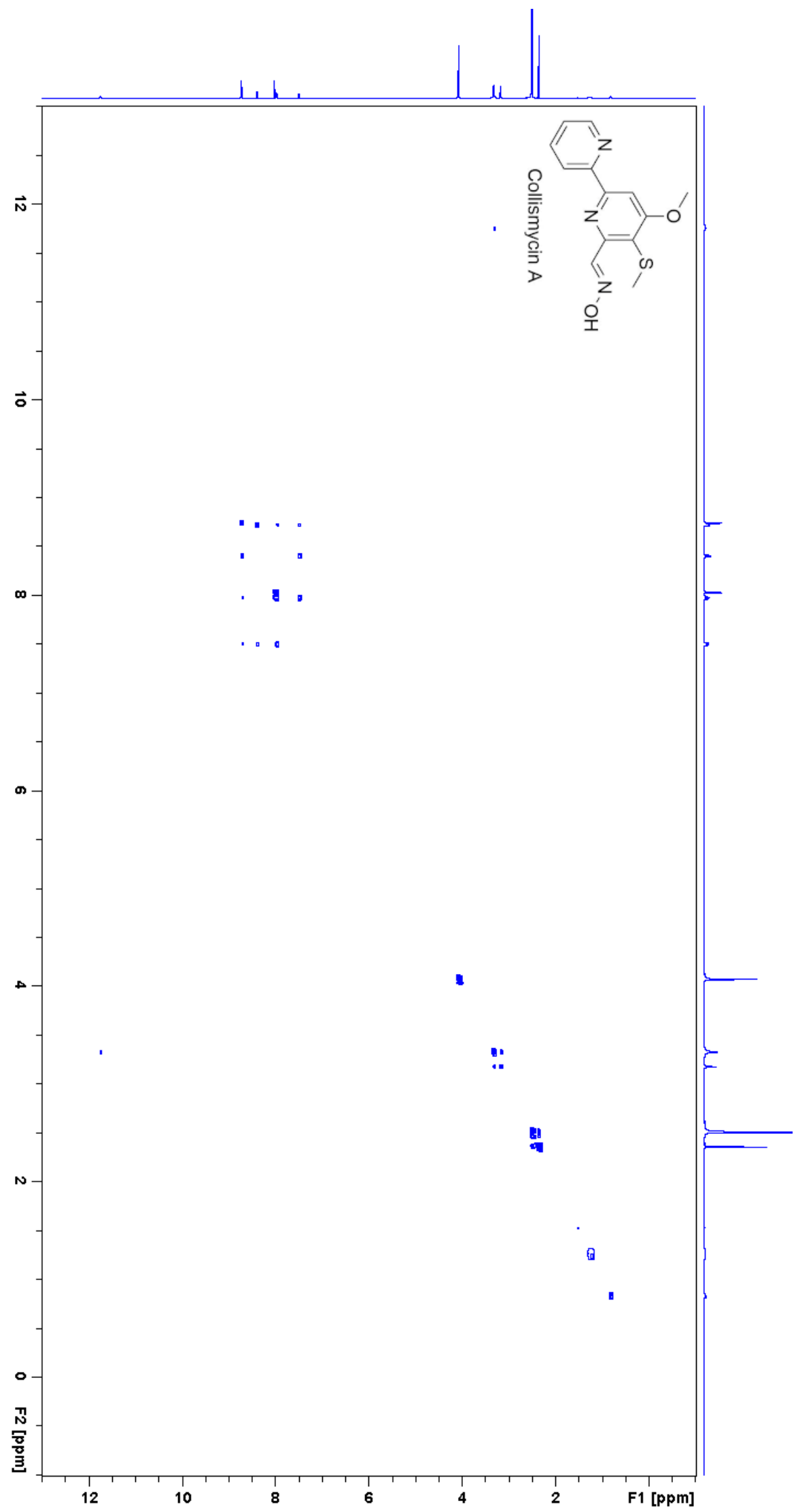

Figure S11: TOCSY Spectrum of Collismycin A (11) in DMSO-d6 


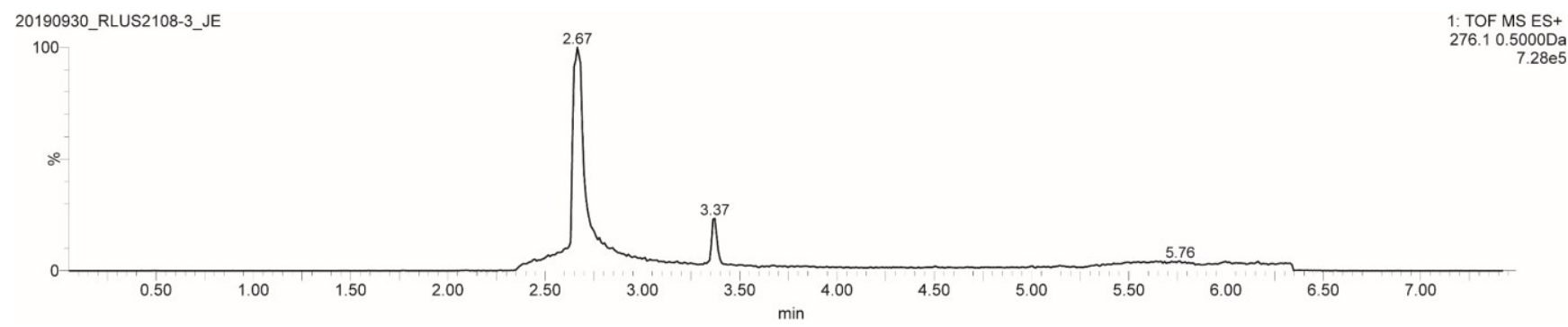

Figure S12: EIC of Collismycin A $\left(\mathrm{m} / z, 276.1[\mathrm{M}+\mathrm{H}]^{+}\right)$

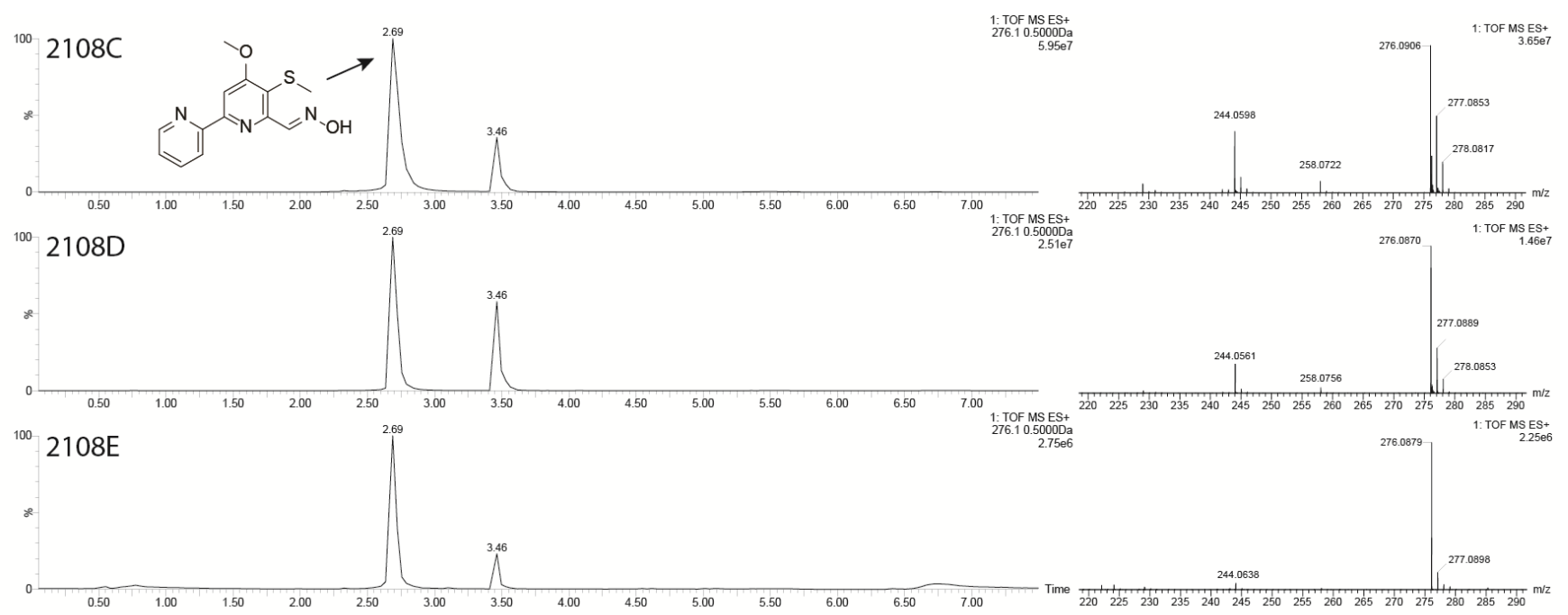

Figure S13: Detection of Collismycin A in Extract Prefractions 
Table S6:Bacterial Strains and Growth Conditions for Biological Evaluation

\begin{tabular}{|c|c|c|c|c|}
\hline Strain Name & Strain Number & $\begin{array}{l}\text { Biosafety } \\
\text { Level }\end{array}$ & $\begin{array}{l}\text { Growth } \\
\text { Medium }\end{array}$ & $\begin{array}{c}\text { Growth } \\
\text { Condition }\end{array}$ \\
\hline \multicolumn{5}{|c|}{ Gram-Positive } \\
\hline Bacillus subtilis & ATCC 6051 & 1 & NB & $37^{\circ} \mathrm{C}$ \\
\hline Enterococcus faecalis & ATCC 29212 & 2 & $\mathrm{BHI}$ & $37^{\circ} \mathrm{C}$ \\
\hline Enterococcus faecium & ATCC 6569 & 2 & $\mathrm{BHI}$ & $37^{\circ} \mathrm{C}$ \\
\hline Listeria ivanovii & BAA-139 & 1 & BHI-A; HTM & $37^{\circ} \mathrm{C} ; 5 \% \mathrm{CO}_{2}$ \\
\hline $\begin{array}{l}\text { Staphylococcus aureus (Methicillin- } \\
\text { Resistant) }\end{array}$ & BAA-44 & 2 & TSB & $37^{\circ} \mathrm{C}$ \\
\hline $\begin{array}{l}\text { Staphylococcus aureus (Methicillin- } \\
\text { Sensitive) }\end{array}$ & ATCC 29213 & 2 & TSB & $37^{\circ} \mathrm{C}$ \\
\hline Staphylococcus epidermidis & ATCC 14990 & 1 & TSB & $37^{\circ} \mathrm{C}$ \\
\hline Streptococcus pneumoniae & ATCC 49619 & 2 & $\mathrm{BHI}$ & $37^{\circ} \mathrm{C} ; 5 \% \mathrm{CO}_{2}$ \\
\hline \multicolumn{5}{|c|}{ Gram-Negative } \\
\hline Acinetobacter baumanii & ATCC 19606 & 2 & TSB & $37^{\circ} \mathrm{C}$ \\
\hline Escherichia coli & K-12 MG1655 & 1 & NB & $37^{\circ} \mathrm{C}$ \\
\hline Klebsiella aerogenes & ATCC 35029 & 1 & NB & $37^{\circ} \mathrm{C}$ \\
\hline Klebsiella pneumoniae & ATCC 700603 & 2 & NB & $37^{\circ} \mathrm{C}$ \\
\hline Ochrobactrum anthropi & ATCC 49687 & 1 & TSB & $37^{\circ} \mathrm{C}$ \\
\hline Providencia alcalifaciens & ATCC 9886 & 1 & TSB & $37^{\circ} \mathrm{C}$ \\
\hline Pseudomonas aeruginosa & ATCC 27853 & 2 & TSB & $37^{\circ} \mathrm{C}$ \\
\hline Salmonella enterica & ATCC 13311 & 2 & NB & $37^{\circ} \mathrm{C}$ \\
\hline Shigella sonnei & ATCC 25931 & 2 & NB & $37^{\circ} \mathrm{C}$ \\
\hline Vibrio cholerae & A1552 El Tor & 2 & TSB & $37^{\circ} \mathrm{C}$ \\
\hline Yersinia pseudotuberculosis & ATCC 6904 & 2 & BHI & $37^{\circ} \mathrm{C}$ \\
\hline
\end{tabular}


Table S7: Biological Activity of Collismycin A (11)

\begin{tabular}{|c|c|}
\hline Organism & $\begin{array}{l}\text { MIC50 } \\
(\mu M)\end{array}$ \\
\hline Bacillus subtilis & 32 \\
\hline Enterococcus faecalis & $>128$ \\
\hline Enterococcus faecium & $>128$ \\
\hline Listeria ivanovii & $>128$ \\
\hline Staphylococcus aureus (Methicillin-Sensitive) & $>128$ \\
\hline Staphylococcus aureus (Methicillin-Resistant) & $>128$ \\
\hline Staphylococcus epidermis & $>128$ \\
\hline Streptococcus pneumoniae & 64 \\
\hline Ochrobactrum anthropi & $>128$ \\
\hline Escherichia coli & 32 \\
\hline Klebsiella aerogenes & 64 \\
\hline Klebsiella pneumoniae & 16 \\
\hline Providencia alcalifaciens & 128 \\
\hline Salmonella enterica & 32 \\
\hline Shigella sonnei & 64 \\
\hline Yersinia pseudotuberculosis & $>128$ \\
\hline Acinetobacter baumanii & 128 \\
\hline Pseudomonas aeruginosa & 32 \\
\hline Vibrio cholerae & 128 \\
\hline
\end{tabular}

\section{MADByTE Processing Steps and Outputs}

\section{Supervised Peak Picking}

Datasets were peak picked manually. The criteria were that peaks displayed a reasonable peak shape, had a discernible maxima, were not in obvious regions of noise, and were above a reasonable threshold. 


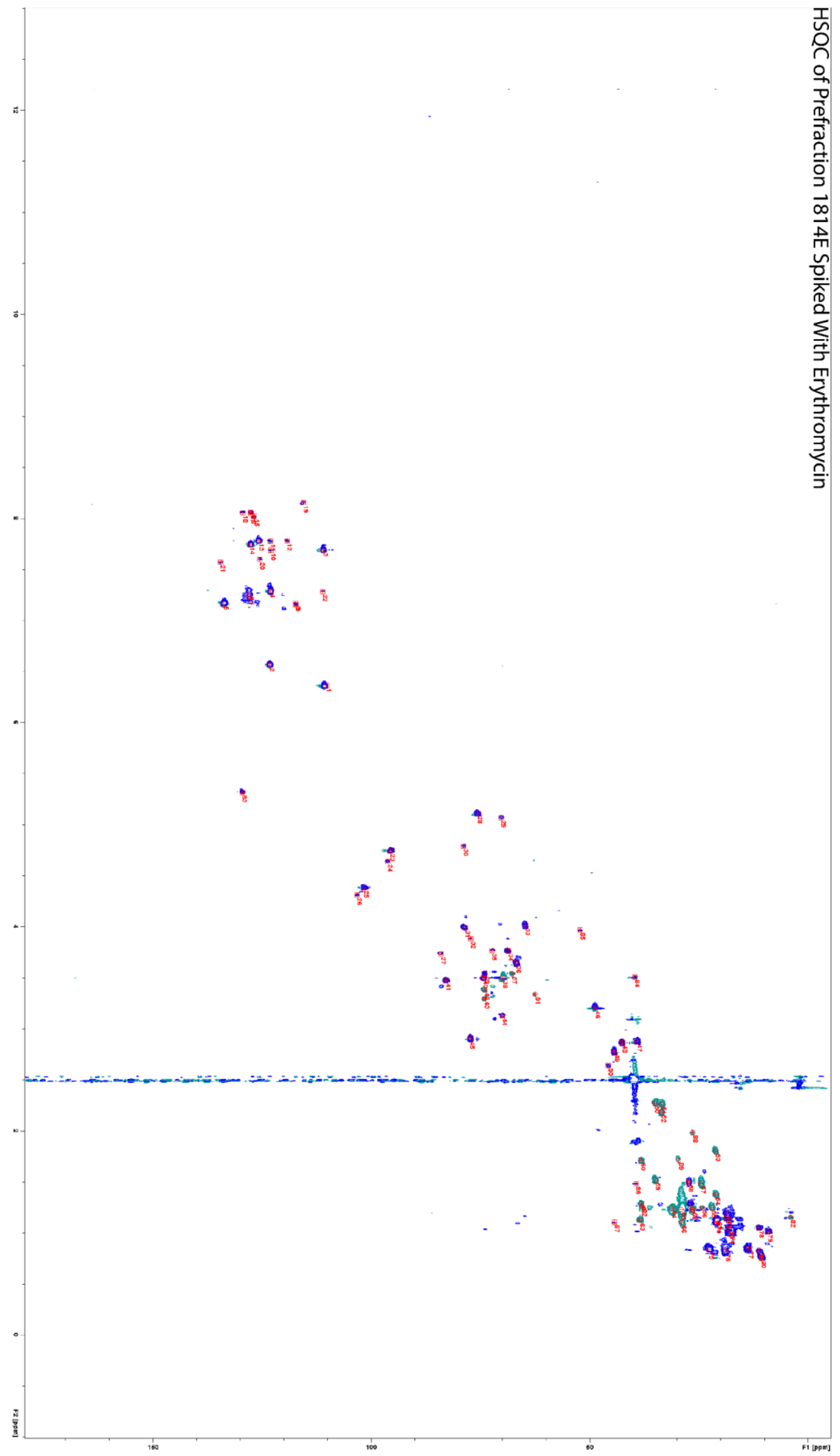

Figure S14: HSQC of Prefraction 1814E Spiked with Erythromycin 


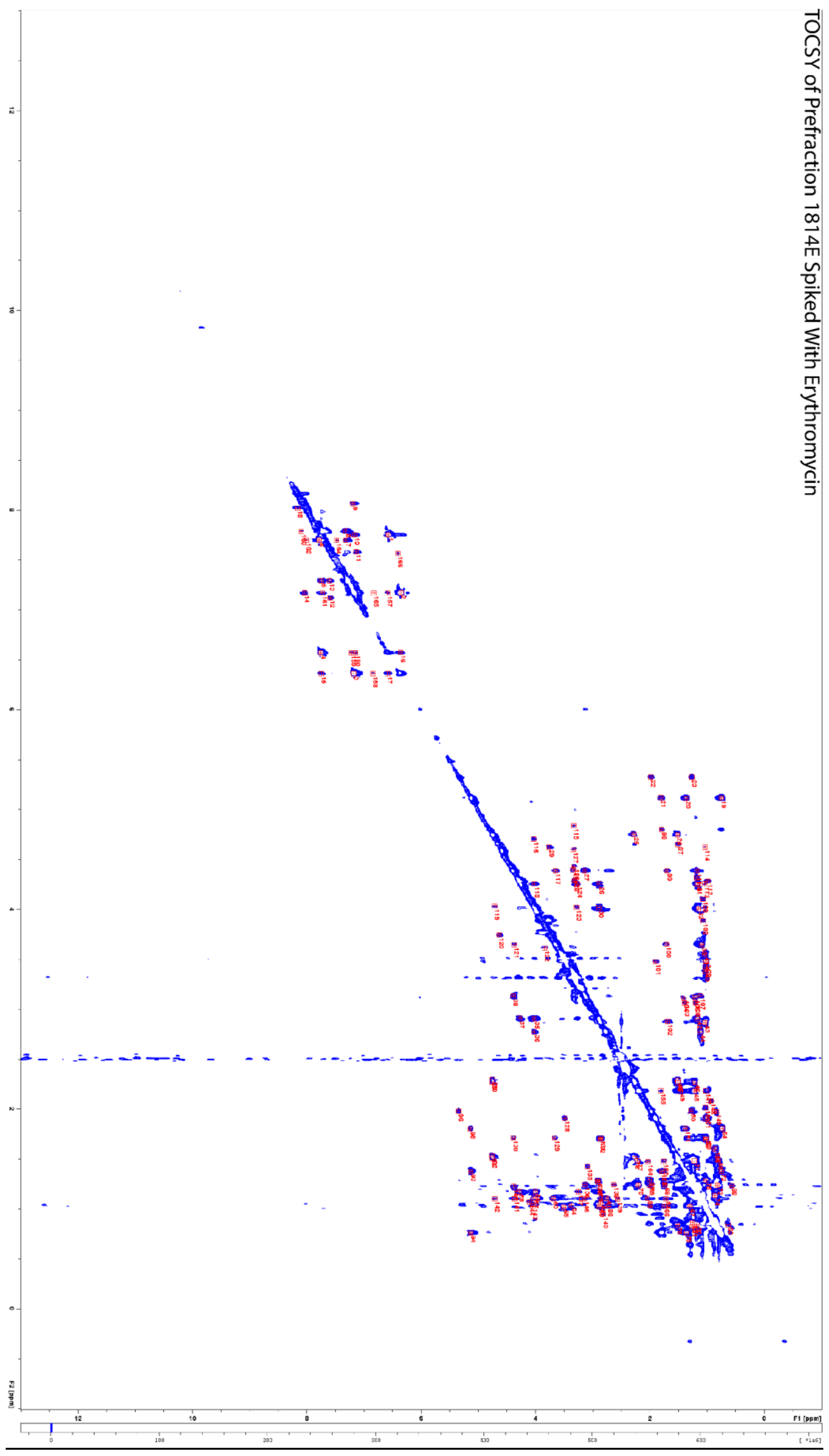

Figure S15: TOCSY of Prefraction 1814E Spiked with Erythromycin 


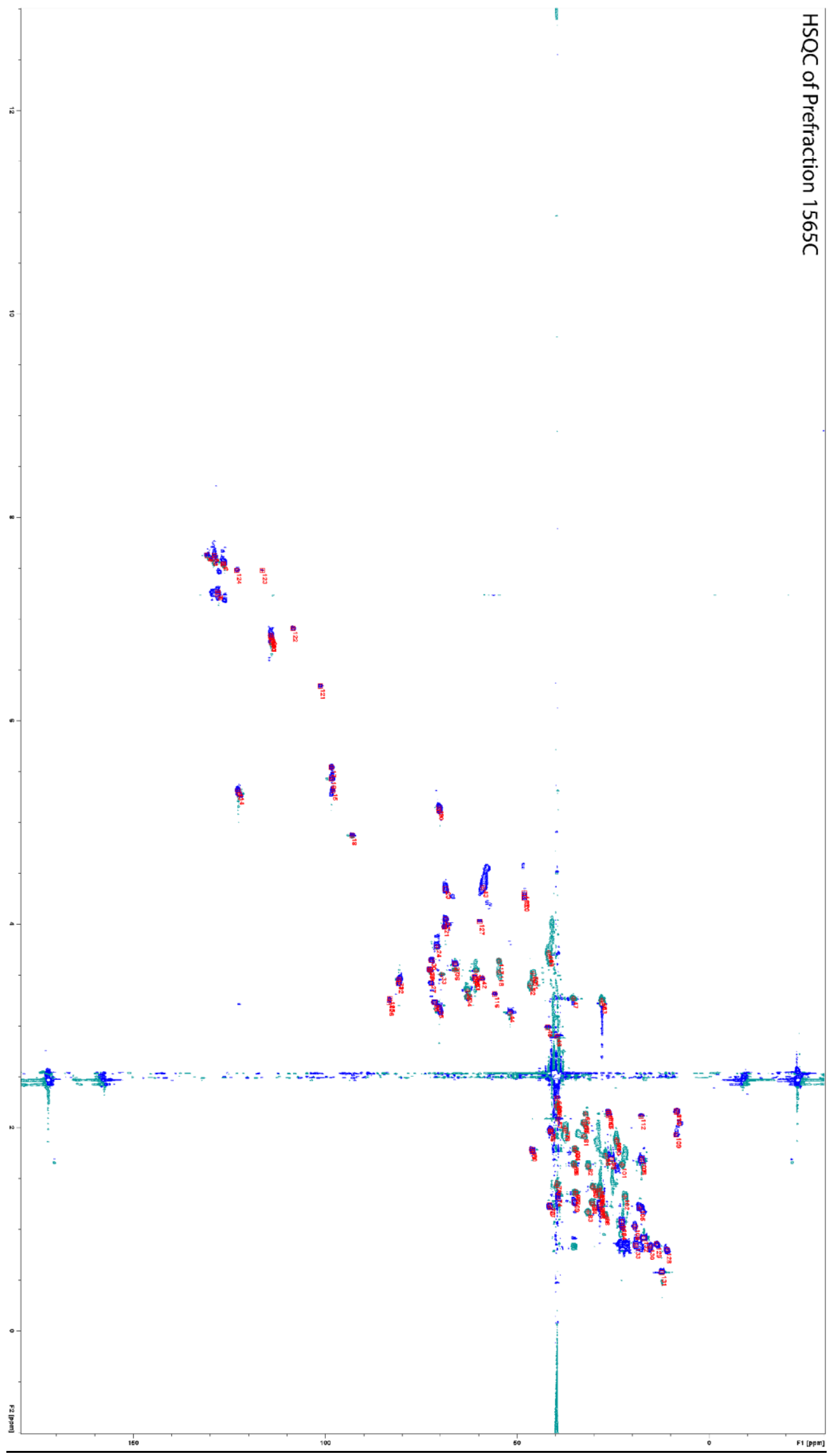

Figure S16: HSQC of Prefraction 1565C 


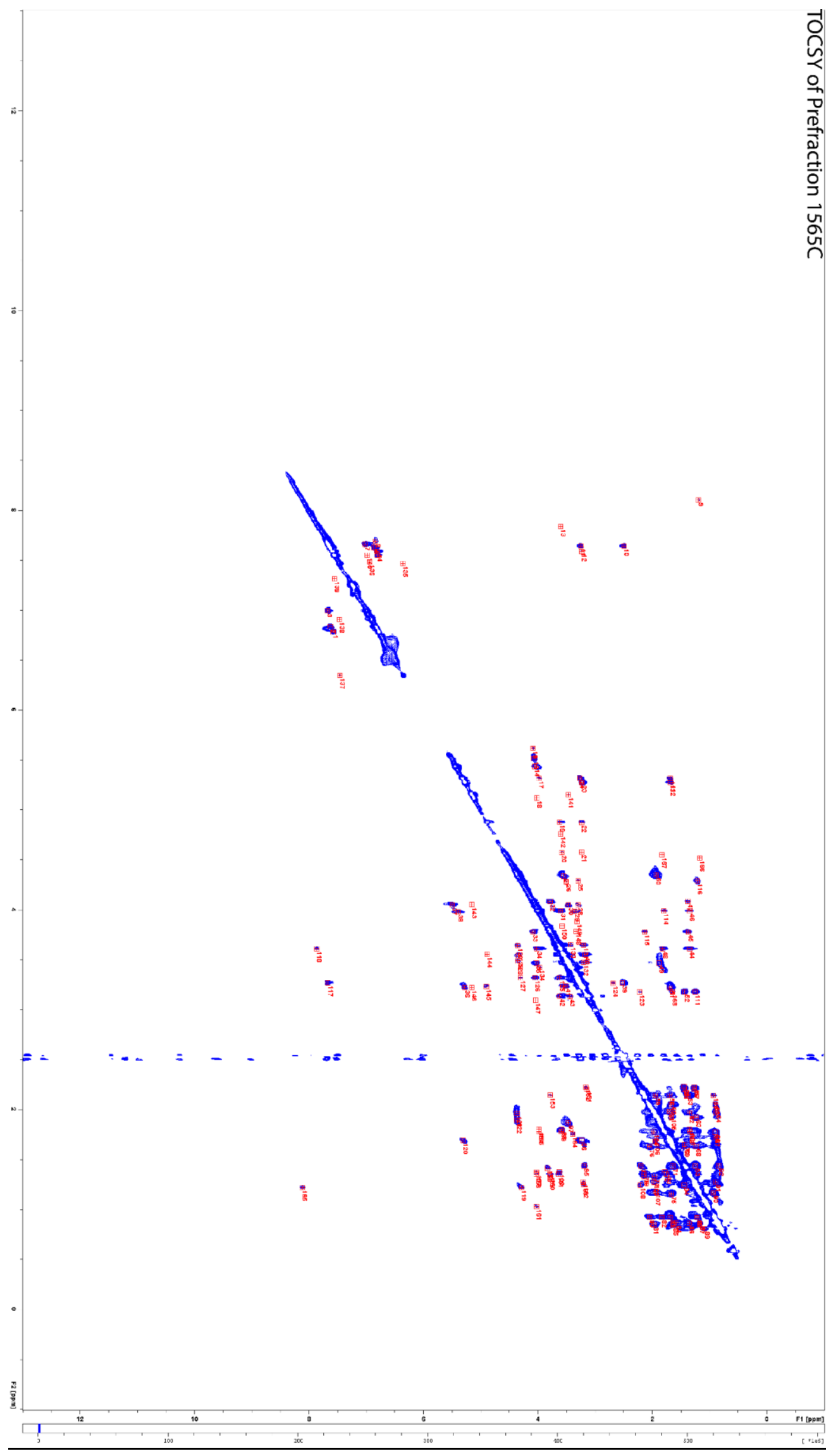

Figure S17: TOCSY of Prefraction 1565C 


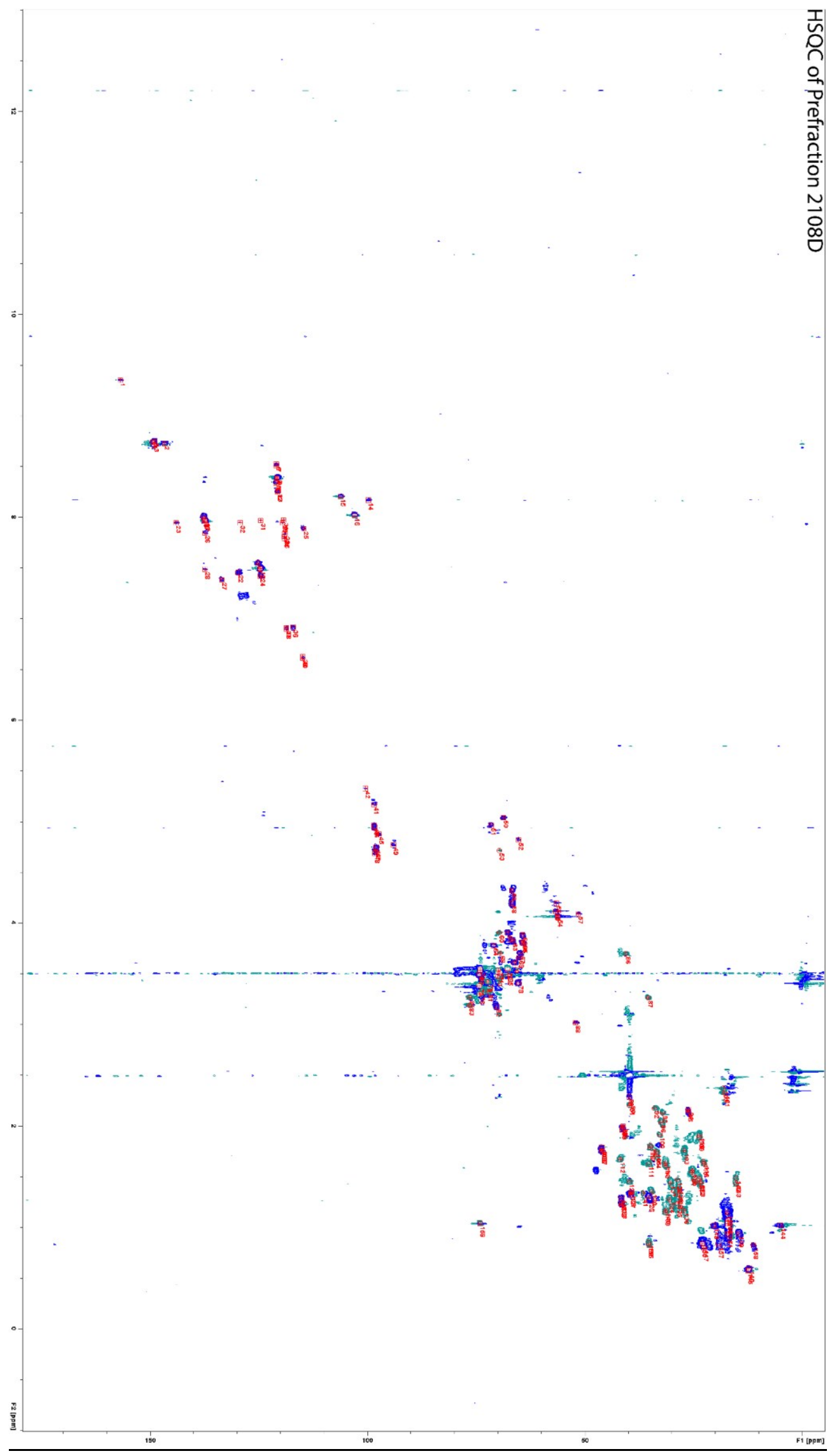

Figure S18: HSQC of Prefraction 2108D 


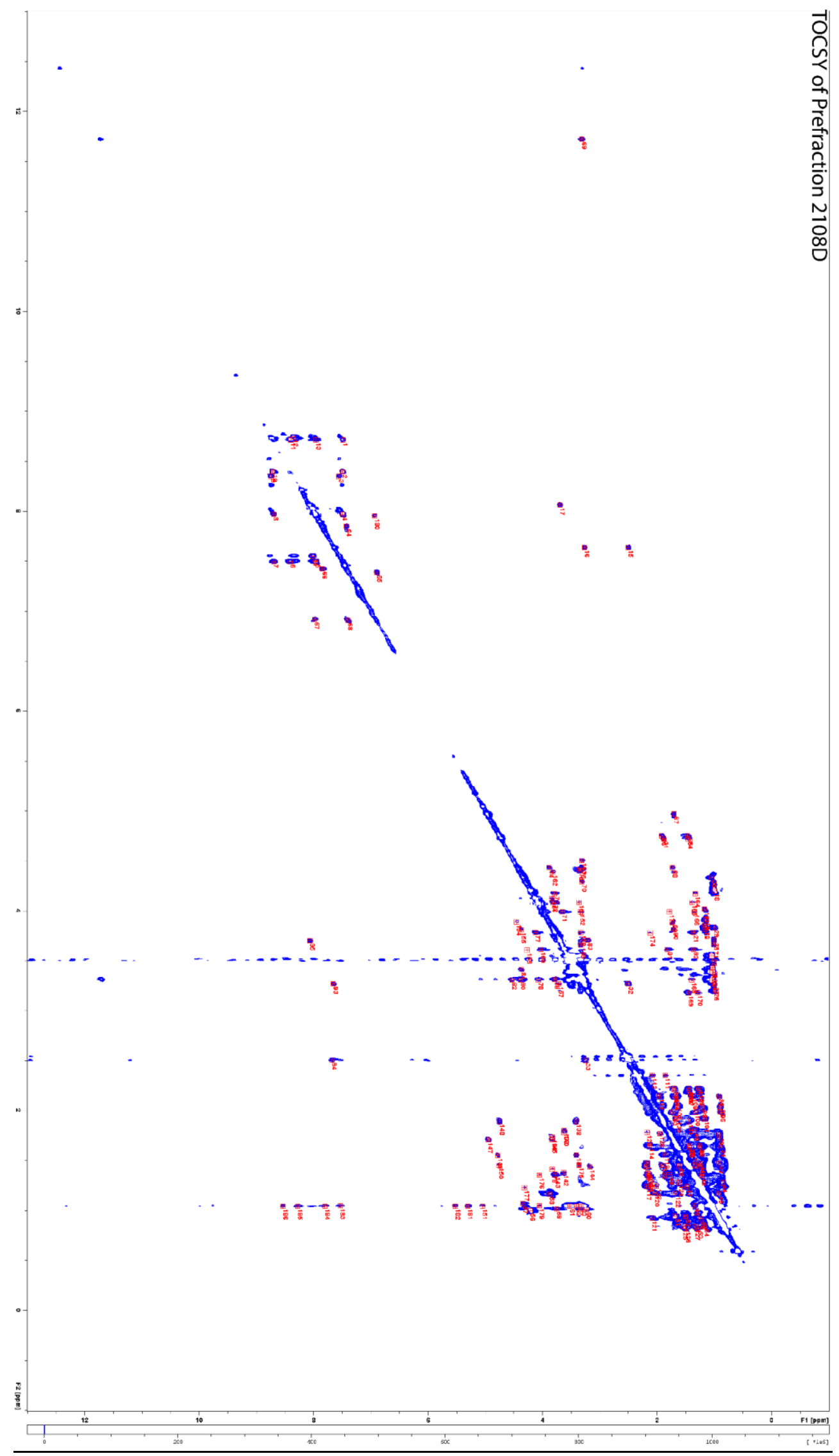

Figure S19: TOCSY of Prefraction 2108D 


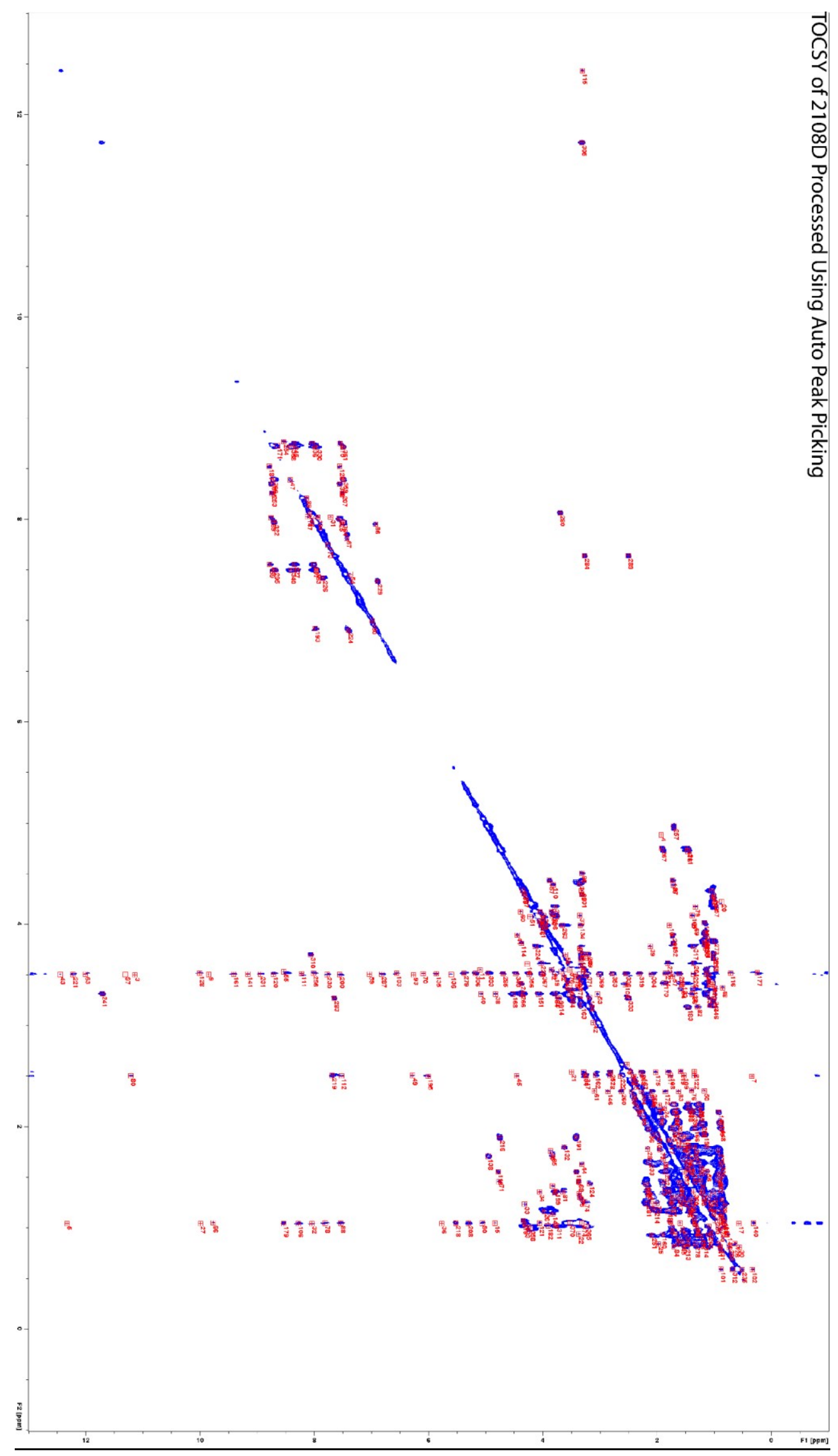

Figure S20: TOCSY of Prefraction 2108D Using Automatic Peak Picking 


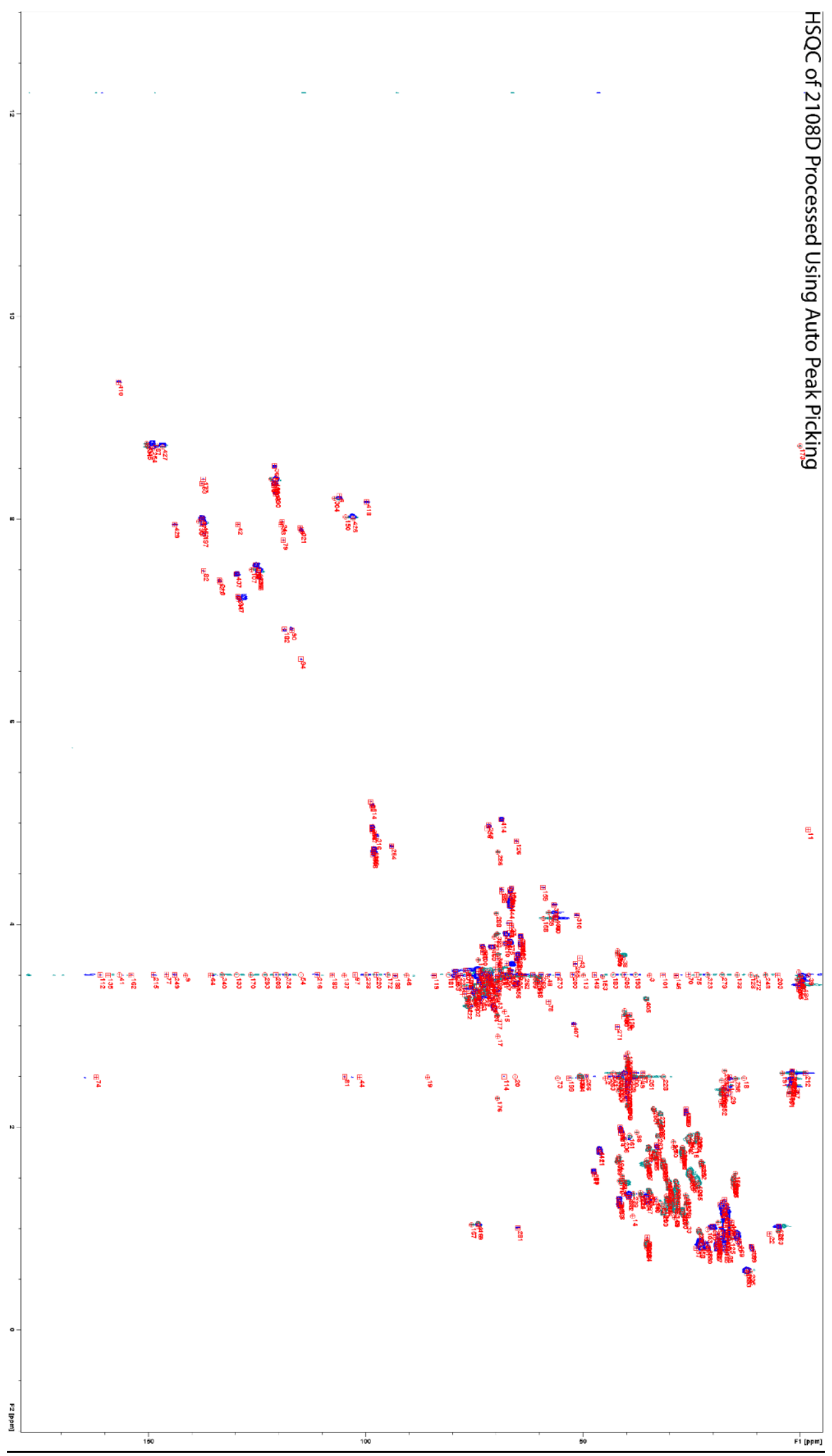

Figure S21: HSQC of Prefraction 2108D Using Automatic Peak Picking 


\section{NMR Data Parsers}

Data parsers have been completed for Topspin txt peak list, Mnova peak table, and ACD Simulated 2D data formats.

\section{HSQC Data Preprocessing}

HSQC data are first filtered to remove peaks in the solvent and water signal regions. In addition, regions with very high disparities between the relative ${ }^{1} \mathrm{H}$ and ${ }^{13} \mathrm{C}$ values are excluded (Figure S22 regions 1,2 , and 3 .

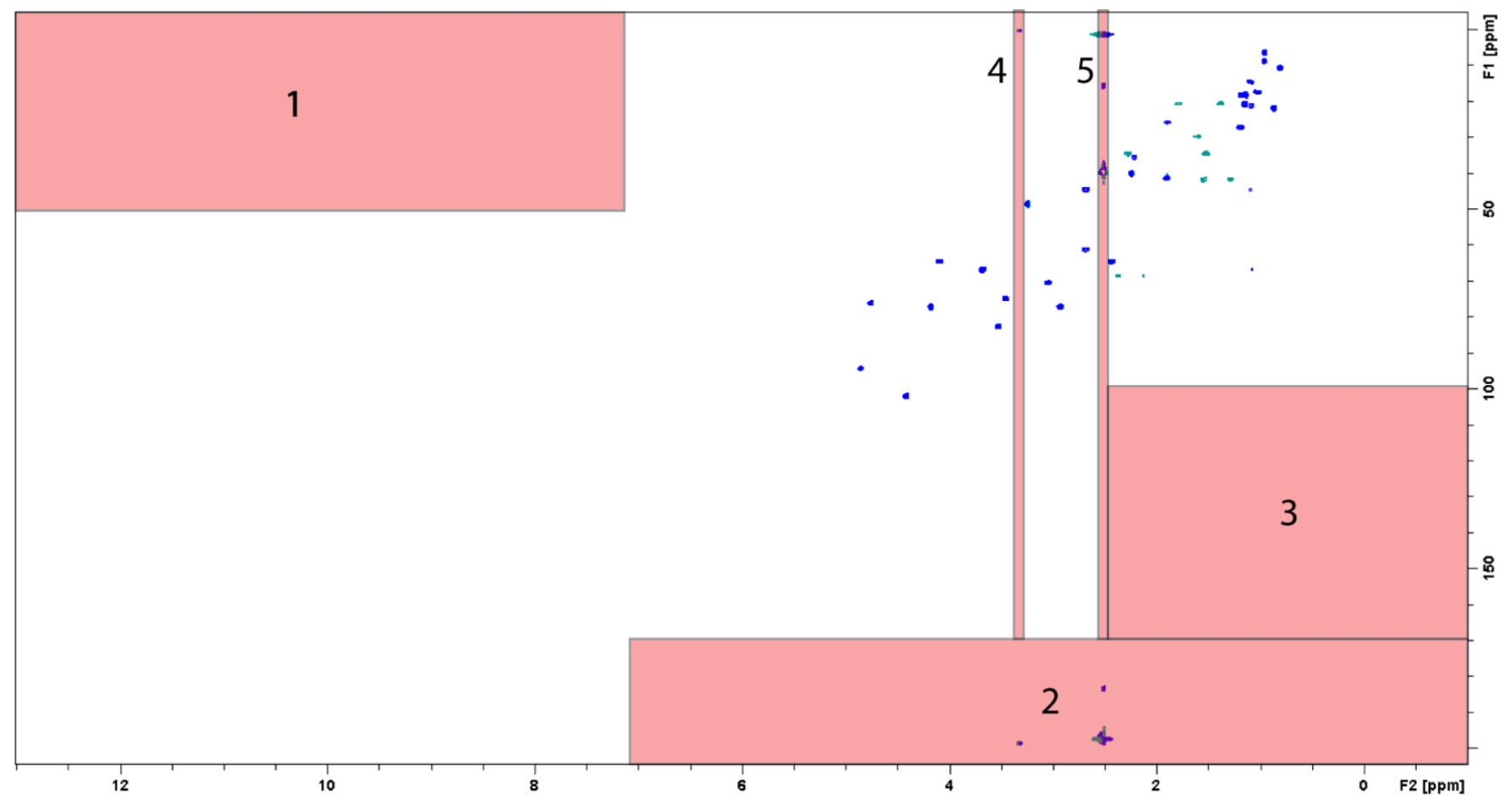

Figure S22: HSQC Datapoint Filtration Zones

Table S8: HSQC Datapoint Filtration Zones

\begin{tabular}{|c|c|c|c|}
\hline Proton Range $(\mathrm{ppm})$ & Carbon Range $(\mathrm{ppm})$ & Reason & Zone \\
\hline $2.48-2.52$ & All & DMSO T1 Noise & 4 \\
\hline $3.28-3.32$ & All & $\mathrm{H}_{2} \mathrm{O}$ T1 Noise & 3 \\
\hline $0.0-2.4$ & $100.0-210.0$ & Extraordinary Shift Disparity & 2 \\
\hline $0.0-7.0$ & $170.0-210.0$ & Extraordinary Shift Disparity & 1 \\
\hline $7.0-13.0$ & $0.0-50.0$ & Extraordinary Shift Disparity & 5 \\
\hline
\end{tabular}

Although HSQC spectra are optimally single resonance datapoints, some multiplet structure is retained without implementation of pure shift experiments. ${ }^{2}$ To address this, after initial data filtration HSQC data points are queried for multiplet structure by finding points within the defined $\mathrm{H} \_$ppm and C_ppm error 
tolerances. Points found within these boundaries are merged as the center of all detected peaks if the phase of the signals match. Users can disable this option in the GUI.

\section{TOCSY Data Preprocessing}

Covariance processing offers a promising alternative to standard processing, as it provides an increase in the resolution in $\mathrm{F} 1 .^{3}$ To evaluate covariance processing, the TOCSY spectrum from azithromycin (3) and an extract prefraction 2108D were processed using the covariance module included in MNova (direct covariance, square root, no filter). ${ }^{4}$ Although this worked well for pure compounds, extract data performed poorly (Figure S23), introducing 'streaking' patterns and signal artefacts that made it harder to accurately pick relevant peaks from the TOCSY spectrum.

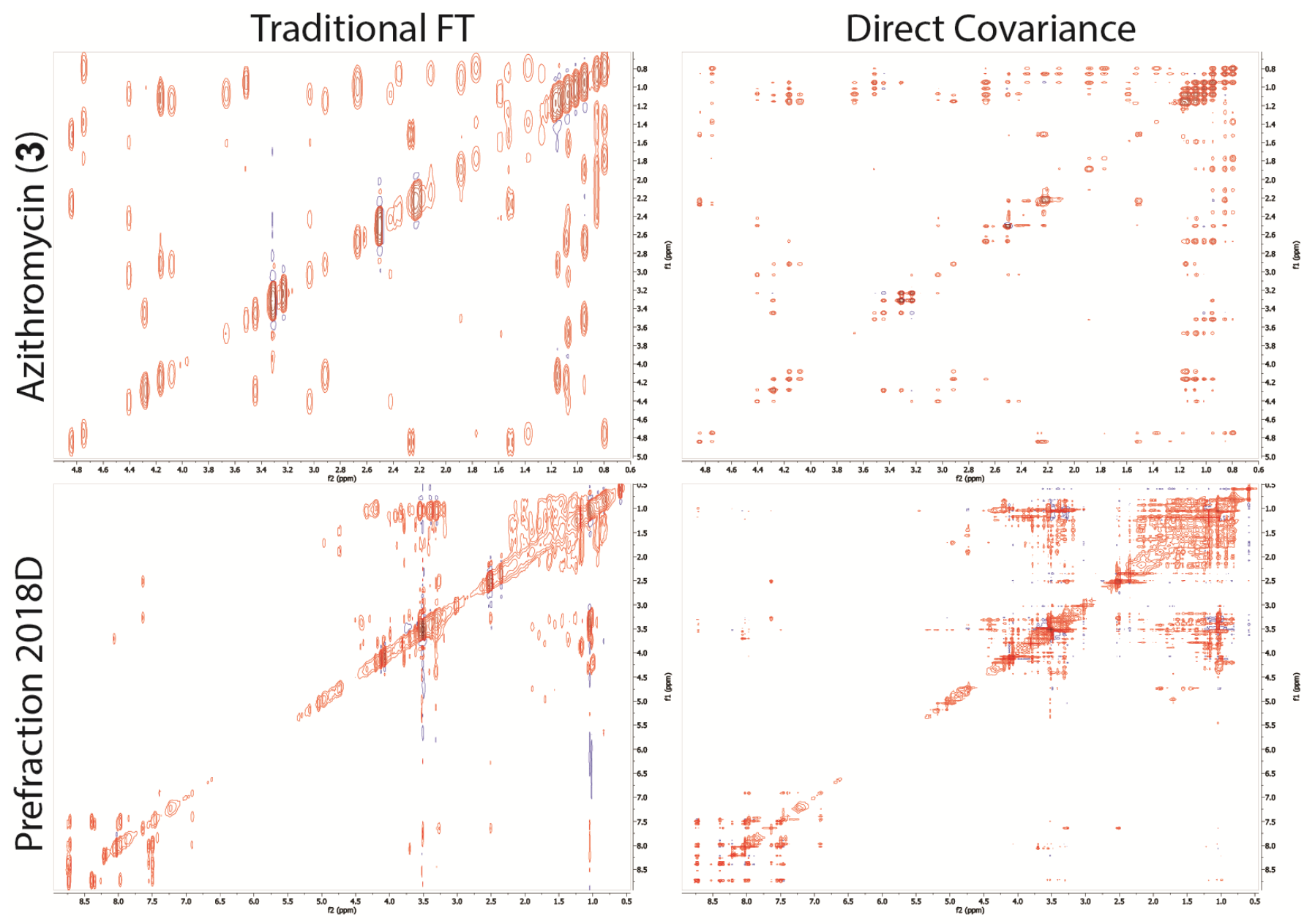

Figure S23: Typical FT vs Covariance Processing for TOCSY Spectra

TOCSY data is filtered by removal of the shielded region in both dimensions $(<2.50 \mathrm{ppm})$, as the overlap in this region is too great to permit accurate peak picking in most cases. Fortunately, data points in this region are often incorporated through their association with deshielded correlations in F2, improving spectral coverage. To gauge the extent to which molecules will have exclusively shielded ${ }^{1} \mathrm{H}$ signals, and therefore would not be amenable to this method, chemical shift predictions were calculated for 27844 compounds in the NPAtlas ${ }^{5}$ using the stereo-aware HOSE code prediction method utilized in NMRShiftDB2. ${ }^{6}$ To remove heteroatom ${ }^{1} \mathrm{H}$ shifts, results were filtered for resonances which were exclusively attached to ${ }^{13} \mathrm{C}$ and would therefore be obtainable via HSQC analysis. Using the provided mean value for each predicted shift, the number of shifts which were above $2.5 \mathrm{ppm}$ were compared 
against the number of possible ${ }^{1} \mathrm{H}$ shifts in each molecule, yielding the percentage of ${ }^{1} \mathrm{H}$ shifts per molecule which were above $2.5 \mathrm{ppm}$ (Figure S24). 19341 compounds (69.46\%) showed more than $25 \%$ of the molecule would have ${ }^{1} \mathrm{H}$ shifts greater than the cutoff of $2.5 \mathrm{ppm}$ and $130(0.4 \%)$ showed no predicted ${ }^{1} \mathrm{H}$ shifts $>2.5 \mathrm{ppm}$ in the entire molecule.

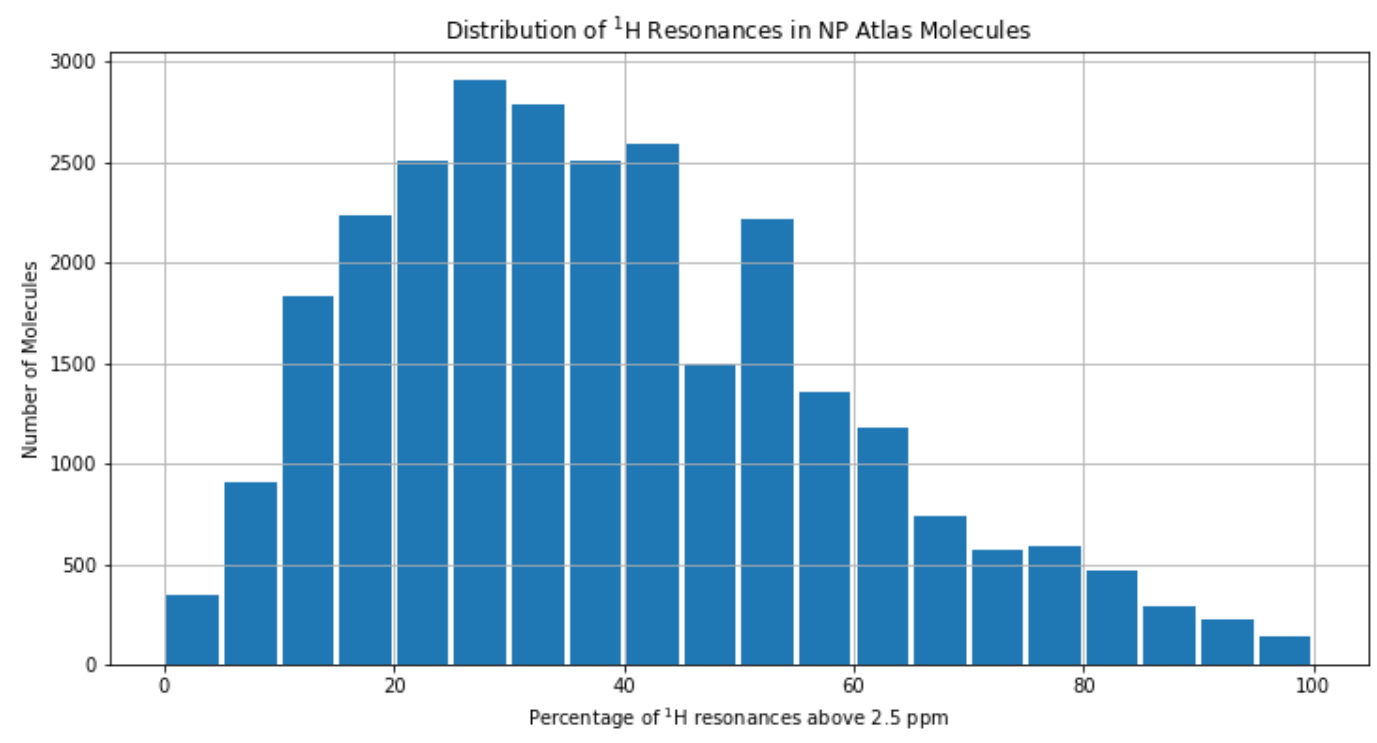

Figure S24: Distribution of Estimated Chemical Shifts in NP Atlas Molecules.

Points identified as self-correlations are also excluded from analysis. Each TOCSY proton signal is then queried to see if a matching proton signal exists within $0.05 \mathrm{ppm}$ in the HSQC spectrum. If no HSQC data point is found, the correlation is removed. By requiring the data points to be present in both the processed TOCSY and HSQC data, false data points arising from reconstruction of non-uniform sample data as well as random noise are eliminated from further analysis.

After data filtration, TOCSY data points are aligned by merging all reported F2 peaks occurring within $0.01 \mathrm{ppm}$. Points in F1 are then aligned to points in F2 within $0.02 \mathrm{ppm}$ and data points outside of this margin are re-queried and merged within a margin of $0.01 \mathrm{ppm}$. This process is designed to take advantage of the increased resolution in the F2 dimension for alignment, eliminating cases of resonance duplication. An overview of the process can be seen in Figure S25. 


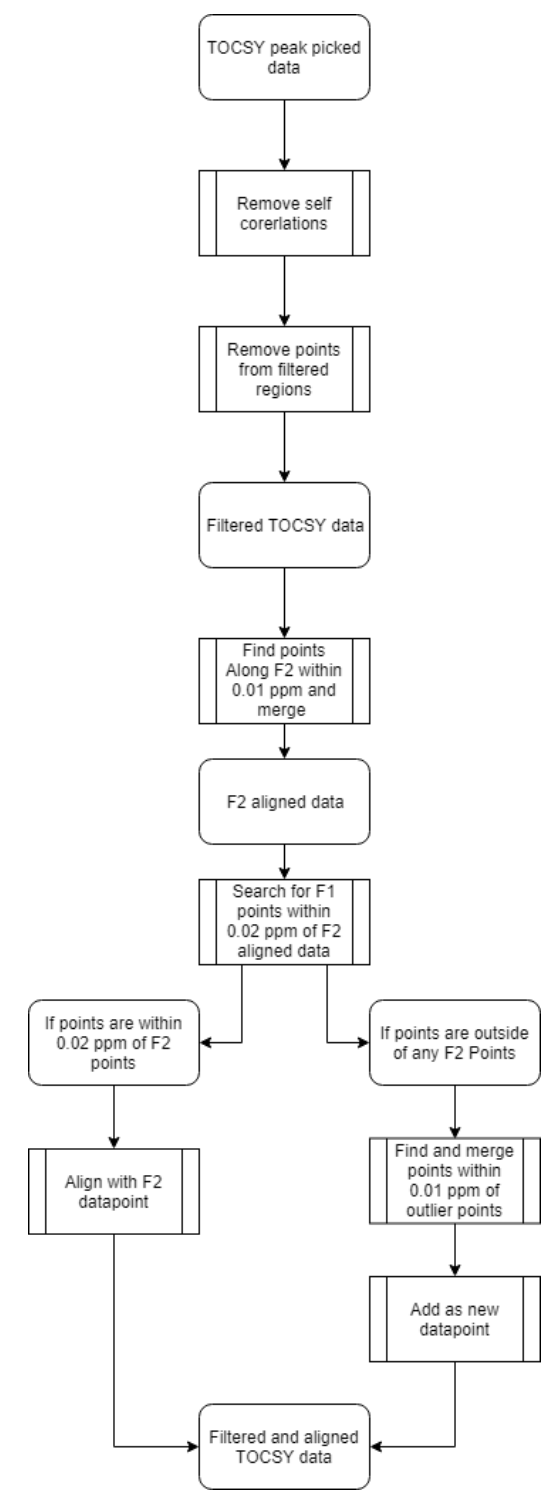

Figure S25:TOCSY Data Point Filtration and Alignment Process

\section{Spin System Networking}

TOCSY correlations are considered valid in the MADByTE algorithm if they share reciprocal correlations or are connected to at least two nodes in the same spin system. A spin system is composed on a set of two or more of these TOCSY correlations, matched to HSQC ${ }^{1} \mathrm{H}^{-13} \mathrm{C}$ correlations. The spin system construction algorithm begins with a directed graph with proton signals as nodes, and edges representing TOCSY cross peak correlations, using the NetworkX package. ${ }^{7}$ Each subgraph of the spin network therefore represents a possible spin system. Nodes with only one edge, representing a single correlation only, are discarded. Edges (and resulting disconnected nodes) in each sub-graph are removed until every spin system meets the validity requirements. Resulting sub-graphs from this process are considered for complete feature construction. Using the erythromycin data as a test case (Figure S26), the imported connections (Figure S26, Panel A) are filtered using these criteria, resulting in more robust (containing no noise signals) spin systems to query the HSQC data (Figure S26 Panel B). 


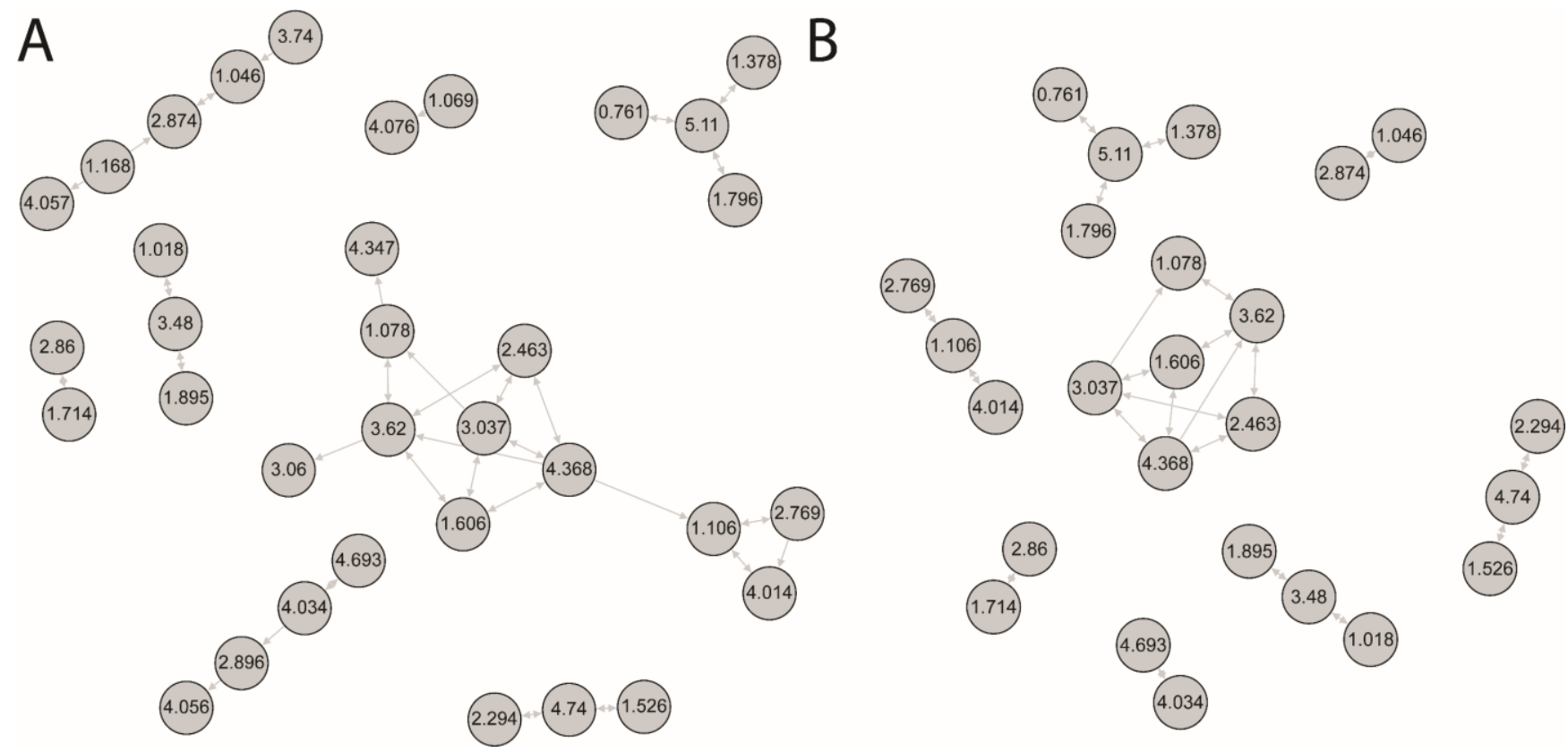

Figure S26: Spin System Construction from TOCSY Data of Erythromycin

Data points in each spin system are then mapped to the HSQC data within $0.05 \mathrm{ppm}$. If two or more HSQC points are within this margin, the closest ${ }^{13} \mathrm{C}$ signal is assigned. In the event that two resonances from the HSQC are equidistant to the TOCSY resonance, both carbon assignments are added as points in the spin system as they cannot be resolved. Resulting spin system features are stored and used in the following step to determine the similarity of spin systems from different samples.

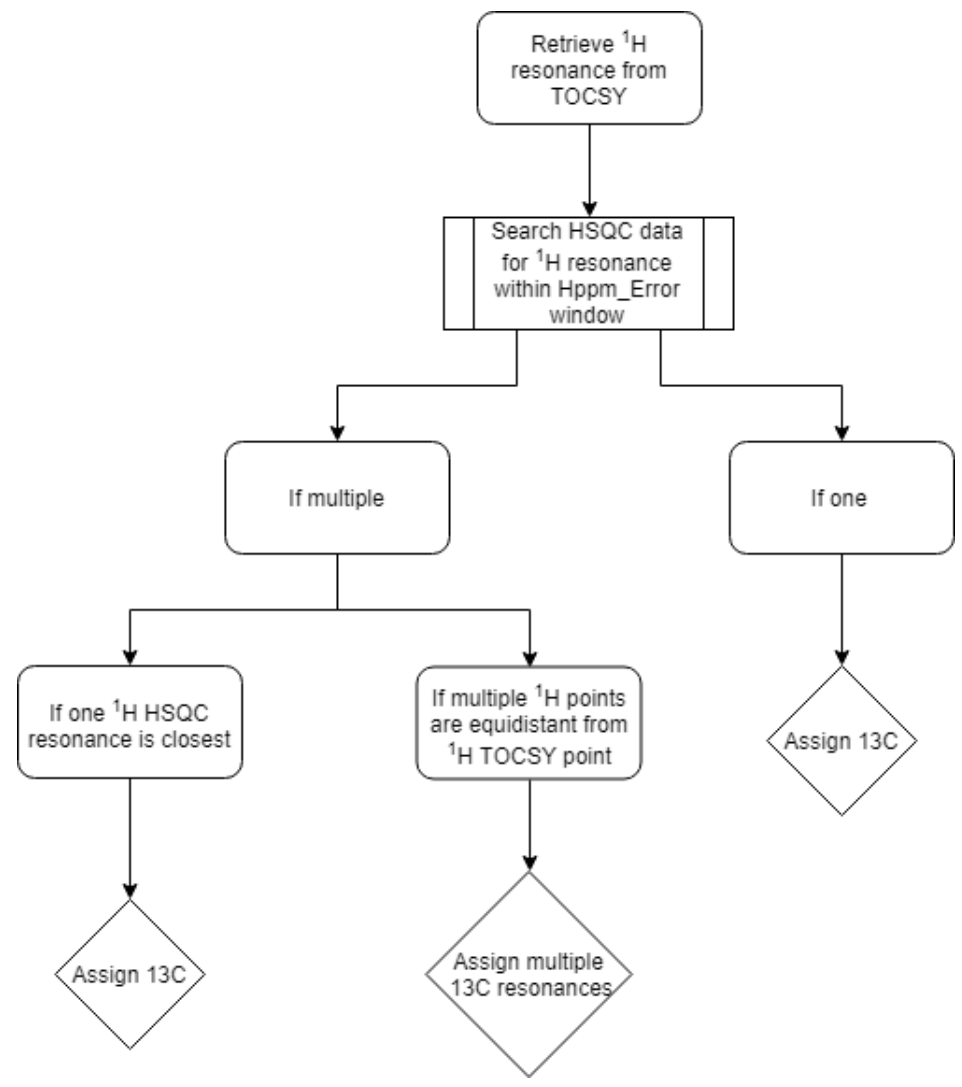

Figure S27:Assigment of HSQC ${ }^{13} \mathrm{C}$ Values to TOCSY Spin System Features 


\section{Similarity Scoring}

Once the spin system construction step is complete, the spin systems must be compared to each other to find the amount of potential overlap between any two systems. We use a similarity ratio to determine the overlap.

To calculate the similarity between two spin systems $s$ and $s^{\prime}$, we consider each spin feature independently. A spin feature consists of a proton resonance signal with a set of one or more carbon signals. The similarity ratio is then given by:

$$
\operatorname{Similarity}\left(s, s^{\prime}\right)=\frac{\text { length }\left(s^{\prime} \cap s\right)}{\text { length }(s)}
$$

where the intersect of two spin systems is determined by finding the number of spin features in system $s^{\prime}$ which overlap with the features in system $s$. Two user defined parameters are employed here; the proton error tolerance and the carbon error tolerance, which default to 0.05 and 0.4 ppm respectively. Two spin features are said to overlap if a proton is found within the given tolerance, provided that proton has at least one carbon which also matches within the given tolerance. As an example, if we have two spin systems [where each spin feature is noted as $\left({ }^{1} \mathrm{H},\left({ }^{13} \mathrm{C}_{1},{ }^{13} \mathrm{C}_{2}, \ldots\right)\right)$ ]

$$
\begin{gathered}
s=\{(1.50,(13.0,30.2)),(3.01,(20.1)),(4.44,(55.5))\} \\
s^{\prime}=\{(1.51,(25.9)),(1.56,(28.9))\}
\end{gathered}
$$

Then the length of the intersect of $s^{\prime}$ in $s$ is 1 , because the first spin features of each system match within tolerance. This would give a similarity ratio of $1 / 3$, or 0.33 . The similarity ratio takes values between 0 (when there is no overlap of two systems) to 1 (when systems are identical or overlap perfectly). For a MADByTE experiment, the similarity ratio between every pair of spin systems is computed and stored in a square, non-symmetrical matrix.

\section{Association Networking and Outputs}

The association networking step provides three different network views, based on spin system correlation scores. In all views, extracts are represented by large nodes (red nodes in Figure S28) In the Full Association Network (Figure S28 A) each spin system feature is represented by a discrete node, with edges defined by the values from the similarity scoring matrix. The Similarity Network (Figure S28 B) provides a simplified view where spin system features with only a single edge (i.e. no matches to other spin system features in the sample set) are removed. Finally, the Hybrid Network (Figure S28 C) collapses individual spin system features in to sets of features shared between samples. These three viewpoints offer three complementary perspectives on the chemical diversity of the sample set. These networks are downloadable as graphML files for ease of use in Cytoscape and Gephi, as well as an exported HTML Bokeh plot for quick viewing. ${ }^{8-10}$ Nodes can be investigated in the Bokeh plots by hovering over each spin system node to view its membership, and nodes can be filtered by size using the a spin system size slider. 


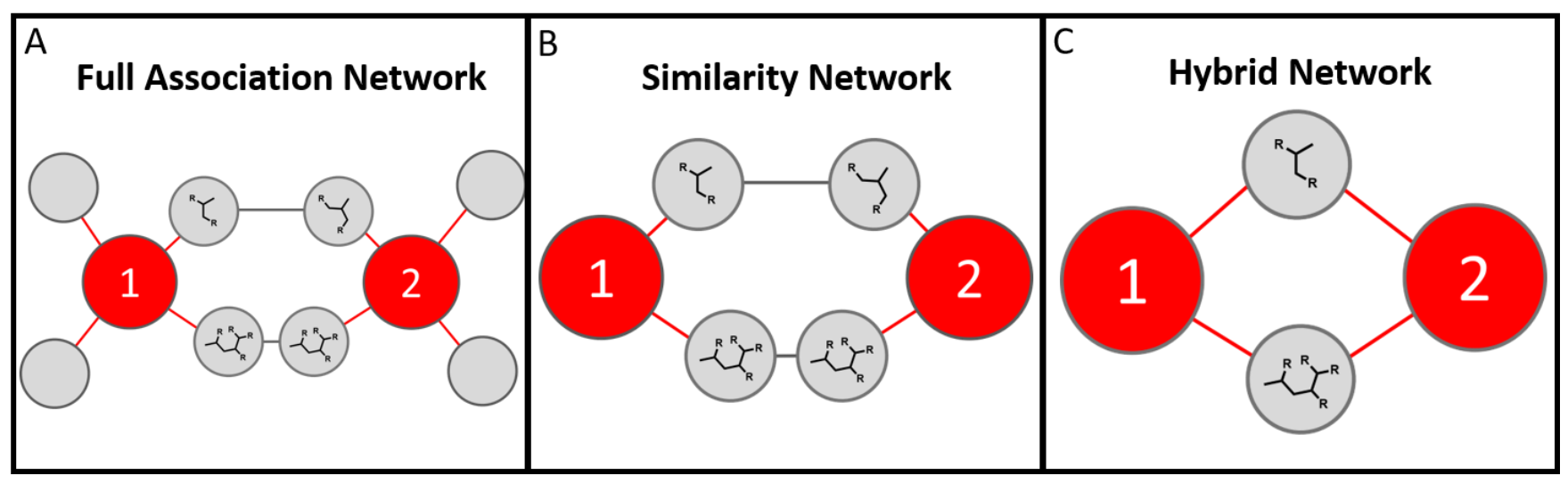

Figure S28: Three Network Output Types from MADByTE

In addition to these three viewpoints, MADByTE also contains a bioactivity layering function which overlays bioactivity data directly onto any one of the three resulting networks. To do this, bioactivity is defined by the user as categorical 'low', 'moderate', and 'high' bioactivity. Using these user defined cutoffs, MADByTE color codes the input network (Figure S29: Panel A) extract ID nodes to reflect the extract's bioactivity (Figure S29: Panel B).

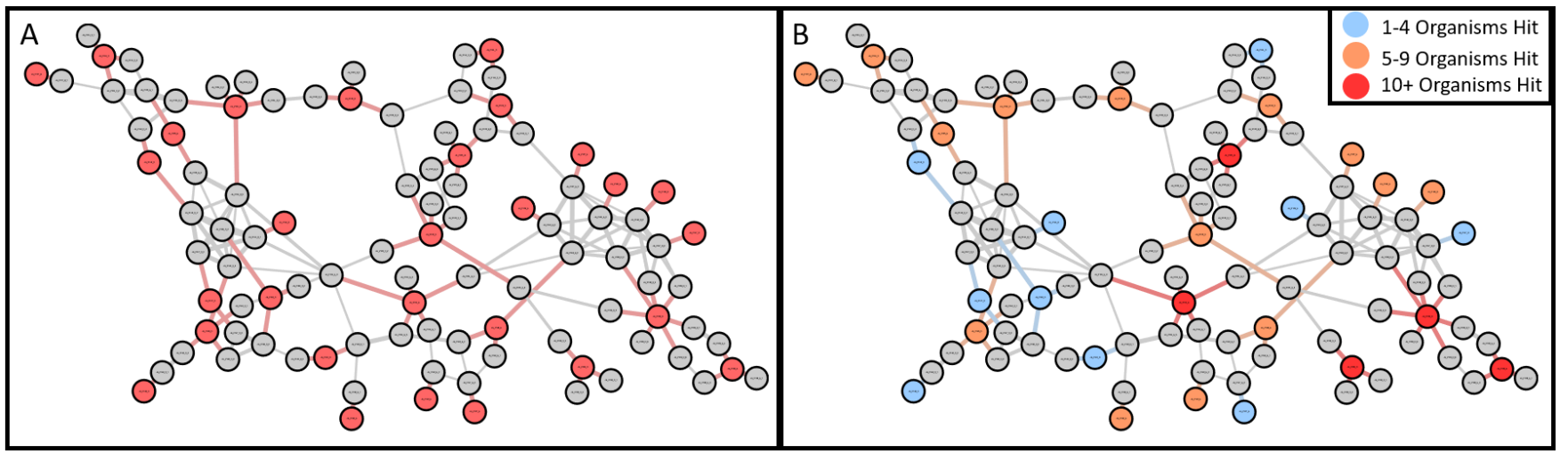

Figure S29: MADByTE Bioactivity Color Coding Example

\section{Dereplication Module}

MADByTE contains a dereplication utility to aid in the recognition of known molecules in a given sample, as well as options for output to allow for simple dereplication via SMART. The native dereplication utility is intended to serve as a local database for quick queries of HSQC resonances of known compounds against the extract data with user definable $\mathrm{H} \_$ppm and $\mathrm{C} \_$ppm cutoffs. Pure compounds can be directly added into the system by first processing with MADByTE and creating a reference file from within the dereplication module, or by providing collections of formatted json files. This enables targeted dereplication of compounds which may be known to the investigator and for which there are reference data. However, there are many cases in which researchers do not have access to reference data. 


\section{Additional MADByTE Resources}

The MADByTE user manual can be found at www.MADByTE.org/resources and contains step by step instructions for all operations, and descriptions of all processing parameters. The MADByTE program is licensed under an MIT software license and is freely available at github.com/liningtonlab/madbyte, and the Linington Lab website (linington.chem.sfu.ca/index.php/resources).

\section{References}

(1) Wong, W. R.; Oliver, A. G.; Linington, R. G. Chem. Biol. 2012, 19 (11), 1483-1495.

(2) Kaltschnee, L.; Kolmer, A.; Timári, I.; Schmidts, V.; Adams, R. W.; Nilsson, M.; Kövér, K. E.; Morris, G. A.; Thiele, C. M. Chem. Commun. 2014, 50 (99), 15702-15705.

(3) Brüschweiler, R.; Zhang, F. J. Chem. Phys. 2004, 120 (11), 5253-5260.

(4) Cobas, C. Stan's Libr. 2014, V (February), 1-8.

(5) Van Santen, J. A.; Jacob, G.; Singh, A. L.; Aniebok, V.; Balunas, M. J.; Bunsko, D.; Neto, F. C.; Castaño-Espriu, L.; Chang, C.; Clark, T. N.; Cleary Little, J. L.; Delgadillo, D. A.; Dorrestein, P. C.; Duncan, K. R.; Egan, J. M.; Galey, M. M.; Haeckl, F. P. J.; Hua, A.; Hughes, A. H.; Iskakova, D.; Khadilkar, A.; Lee, J.-H.; Lee, S.; Legrow, N.; Liu, D. Y.; Macho, J. M.; McCaughey, C. S.; Medema, M. H.; Neupane, R. P.; O’Donnell, T. J.; Paula, J. S.; Sanchez, L. M.; Shaikh, A. F.; Soldatou, S.; Terlouw, B. R.; Tran, T. A.; Valentine, M.; Van Der Hooft, J. J. J.; Vo, D. A.; Wang, M.; Wilson, D.; Zink, K. E.; Linington, R. G. ACS Cent. Sci. 2019, 5 (11) 1824-1833.

(6) Kuhn, S.; Johnson, S. R. ACS Omega 2019, 4 (4), 7323-7329.

(7) Hagberg, A. A.; Schult, D. A.; Swart, P. J. Exploring network structure, dynamics, and function using NetworkX https://networkx.github.io/.

(8) Bokeh Development Team. Bokeh: Python library for interactive visualization http://www.bokeh.pydata.org.

(9) Shannon, P.; Markiel, A.; Ozier, O.; Baliga, N. S.; Wang, J. T.; Ramage, D.; Amin, N.; Schwikowski, B.; Ideker, T. Genome Res. 2003, 13, 2498-2504.

(10) Bastian, M.; Heymann, S.; Jacomy, M. Gephi: An Open Source Software for Exploring and Manipulating Networks https://gephi.org/. 